\title{
Numerical Simulations of Magnetic Dipole over a Nonlinear Radiative Eyring-Powell Nanofluid considering Viscous and Ohmic Dissipation Effects
}

\author{
R. Sajjad, M. Mushtaq, S. Farid, K. Jabeen $(\mathbb{D}$, and R. M. A. Muntazir \\ Department of Mathematics, University of Engineering and Technology, Lahore 54890, Pakistan \\ Correspondence should be addressed to K. Jabeen; kanwaljabeen44@yahoo.com
}

Received 20 May 2021; Revised 23 June 2021; Accepted 20 August 2021; Published 9 September 2021

Academic Editor: SIvasankaran Sivanandam

Copyright $\odot 2021$ R. Sajjad et al. This is an open access article distributed under the Creative Commons Attribution License, which permits unrestricted use, distribution, and reproduction in any medium, provided the original work is properly cited.

\begin{abstract}
This research work interprets the influences of magnetic dipole over a radiative Eyring-Powell fluid flow past a stretching sheet while considering the impacts of viscous and ohmic dissipation that produce a quite illustrious effect due to the generated magnetic dipole. This whole analysis is characterized by the effects of steady, laminar, and incompressible flow. The highly nonlinear and coupled partial differential equations (PDEs) are remodeled into a system of nonlinear ordinary differential equations (ODEs) by utilizing reliable and nondimensional parameters leading to the momentum, thermal, and concentration equations, that are computationally solved using $b v p 4 c$ on MATLAB, and "dsolve" command on MAPLE software, in the companionship of boundary conditions. The physical constraints such as viscous and ohmic dissipation and many other sundry parametric effects are sketched with their ultimate effects on fluid flow. For the sustenance of this research with the prior work and in collaboration with the below mentioned literature review, a comprehensive differentiation is given, which defines the sustainability of the current work. The Buongiorno nanoliquid model elaborates the thermophoresis and Brownian features that are deliberately scrutinized within the influence of activation energy. Also, the skin friction coefficient, Nusselt number, and Sherwood number are illustrated in tables. The skin friction coefficient decreases with a rise in the ferromagnetic interaction parameter as well as the Hartmann number, whereas the Nusselt number and Sherwood number show variation for varying parameters. It can be observed that Eyring-Powell fluid intensifies the rate of heat and mass transfer.
\end{abstract}

\section{Introduction}

From the past few years, the fluids eventuated as most significant in almost all the engineering applications, industrial, as well as in technological approaches because of their exclusive property of having no fixed shape and a deforming nature due to external pressure (forces). Also, fluids are further categorized in Newtonian and nonNewtonian fluids having wide petitions in paper production, food factory, paint manufacturing, polymer industry, chemical processing, and profound and boundless applications in manufacturing. The phenomenon of heat transfer has now become a tremendous point to research purposes due to its inflated requirements in many industrial processes such as the cooling process of electronics, lumped system analysis, nuclear reactor insulation, climate control, heat exchangers, architecture, and solar collector. The stretching of a sheet is a significant phenomenon in polymer industry. The fluid flow that occurs due to availability of stretching sheets was firstly explored by Crane [1] and then outstretched by many authors. So far studies about the impacts of the magnetic field over a stretchable sheet show gigantic significance because of its wide petitions in many engineering sites including geophysics, production of paper, to purify crude oil, banishment of plastic sheet, sustained casting, or spinning of fibre due to stretching surface. The outcomes of the external magnetic field in the presence of MHD flow over a stretching sheet were investigated by Pavlov [2] in detail. Gupta and Gupta [3] investigated heat and mass transmission in the presence of the stretching sheet with suction/blowing and presented numerical solution of the boundary layer equation with variation in different 
parameters. In [4], Rasool et al. studied heat and mass transfer for nonlinear stretching surface using Darcy-Forchheimer relation.

As fluids have common utilization for the phenomenon of heat dissipation as heat transporter, the combined effects of magnetic dipole with fluid is proved influential in constraining the temperature and momentum boundary layers. The turbulent effects in the flow field diminished gradually through magnetic dipole. The effect of magnetic dipole summarizes the restriction of one of the two forms, the closed loop of electric current or couple of poles, when bulk of source diminishes to zero profile, in the case of constant magnetic moment. With an incremented distance from the source, the magnetic source surrounded by the magnetic field looks progressively like the field of magnetic dipole. Kroh and Felderhof [5] scrutinized the impacts of electric and magnetic field on the dynamics of suspension of fluid's particle sustained with electric and/or magnetic dipole effect.

The low thermal conductivity is an utmost limitation in the extension of energy efficient heat transfer fluids needed in many industrial applications. Based on the emanated demand in several industries, the devices involving heat exchange be necessitated with compact size, weighing very little and highly efficient. In other words, such devices critically visualized for uttermost thermal efficiency. Quite merely, the conventional fluids such as oil, water, and ethylene glycol exhibit low thermal conductivity. Keeping in mind the property of high thermal conductivity in fluids, there arises a need of time to design such fluids which exhibit sensationally high thermal conductivity. Numerous attempts have been made through many experiments and minichannels, by means of extended surfaces to increase the heat transmission rate, but found quite incredible that a suspension of small quantity of nanoparticles in the conventional fluids enhances the thermal conductivity to a much better extent. Such fluids are called nanofluids. It refers to nanoparticles in base fluid. The name of nanofluid was firstly introduced by Choi and Eastman [6]. In [7], Rasool et al. considered second-grade nanofluid towards a vertical Riga plate for the Buongiorno nanoliquid model. Several models of non-Newtonian fluids are found involving Maxwell, Williamson, Oldroyd-B, Walter-B model, power law model, etc., but these models did not fully describe all the exclusive features of rheological fluids. For more comprehension of non-Newtonian fluids, the Eyring-Powell nanofluid was firstly proposed by Eyring and Powell in 1944.

The Eyring-Powell model is one of the non-Newtonian models developed for scientific research and is very complex in nature. Yet, this model has many advantages over the other non-Newtonian formulations, including simplicity, easy to compute, and physical robustness. Following are the two main properties of Eyring-Powell fluid that make it unique and significant over some other non-Newtonian models.

(i) It has been derived from kinetic theory of liquids instead of empirical relation

(ii) The model easily reduced to a Newtonian model, just for higher and lower shear rates
Few are the examples of this fluid model including slurries (a mixture of cement or clay with water), tooth paste, human blood, etc. The model belongs to the fluid model containing three constants. This model is competent to exhibit a nonzero viscosity in the surrounded domain at the surface as well as in the ambient fluid discussed in [8].

Viscous dissipation is a phenomenon to endure the work done mutated into heat, a conversion of kinetic into internal energy. The work is done by the fluid on juxtapose layers in the companionship of shear force. The propensity of viscous dissipation effects on the temperature profile will be liable to influence the heat transfer. Consequently, during the high extrusion in polymer processes or especially in blowing existence, the temperature distribution enhanced. In [9], Healy et al. worked for the successive progress of the viscous dissipation function as a concept of velocity gradient where the whole approximation is based on power loss in fluid dynamics. So far, a lot of related work had been done in this regard. Ohmic dissipation of heat is beneficial in heating the liquid edibles accommodating large particulates. For instance, soups, cutting sliced fruits, other heat sensitive fluids, and also helpful in the treatment of proteinaceous foods. Potentially, it has applications in blanching, discerning of starch gelatinization, etc.

The accommodation of minimum energy to initiate the chemical reaction is because sometimes, during the chemical reaction, the molecules collaborate with adequate energy. The debasement of bonds is amenable by two significant energies, the kinetic and potential. At this particular time, the loss in kinetic energy and unsustained collisions requires a compatible amount of energy to activate the molecules for the reaction, and this leads to the concept of activation energy. This activation energy is vigorously used in the field of biochemistry, geothermal industries, hydrodynamics, etc. In [10], Mazzotti et al. explored the work for thermal dependency of viscosity sufficiently explained by activation energy and Arrhenius model along with the distinctive function of the concentration volume fraction. In [11], Saghir and Eslamian highlighted the effects of net transportation of heat by using the concept of activation energy of self-diffusion, and the obtained solution were more accurate as compared to the solutions we get while approximating it by the idea activation energy of viscous flow. Babu and Sathian [12] probed to analyze water accompanying activation energy through carbon nanotubes. Javed et al. [13] explored his work for the boundary layer (BL) flow using non-Newtonian characterized fluid in the presence activation energy over a stretching sheet. See regarding references to further study on activation energy and chemical reaction.

Thermal radiation comes out when technological methodologies are enacted at high value of temperature. It is the emanation of electromagnetic waves from the corresponding fluid at a temperature that is higher than that of absolute zero. So, when there occur small changes in the temperature gradient, it is advantageous to use the concept of linear thermal radiation. But if there are disastrous changes occur in the temperature gradient, then the nonlinear thermal radiation is useful to consider. For example, the case of rocket propulsion and nuclear reactor. Hayat et al. [14] had a detailed work on 3- 
dimensional BL flow of viscoelastic nanofluid using nonlinear thermal radiation, and the condition for the requirement of zero mass flux was executed. The sensations associated with the chemical reaction have prodigious contributions in many chemicals-based industries. Many researchers came in market and gave their tremendous efforts on the impacts of the chemical reactions on different problems. Narayana and Babu [15] explored that how non-Newtonian fluids are influenced by the chemical reaction for a stretching sheet. Related reviews for this analysis are referred [16-21] for further knowledge. In [22], Azhar et al. discussed the effects of viscous dissipation influenced by chemical reaction and considered the stagnation point flow. In [23], Jabeen et al. gave the idea about influencing MHD flow in porous media, and the solution were obtained by using DTM (differential transformation method) and VIM (variational iteration method) approach. Similar results about magnetic dipole, viscous dissipation, and radiation are cited for further study in below references [24-30].

Motivated by the aforesaid literature review, this current pattern has a superficial role of Eyring-Powell non-Newtonian fluid over the stretching surface because of its wide petitions in the production of electronic devices and many medical apparatus, glass fibre, and filaments. Our model basically describes the exceptional viscous and ohmic dissipation effects subjected to magnetic dipole of chemically reacting fluid so this research work is quite advantageous in the field of paper production and manufacturing of plastic. While, in areas of quantum mechanics, aerospace engineering, and space technology, the role of this work is useful due to magnetic dipole. The surface (sheet) is stretchable linearly. No doubt, local similarity transformations with nondimensional expressions are used to transmit the highly nonlinear coupled governing equations into simple ODEs and are then resolved. The solutions are extracted on the basis of numerical strategy by using the reliable algorithm of bvp4c on MATLAB and MAPLE dsolve, and then, the influences of different active parameters on velocity, temperature, and concentration profiles are contemplated, and ultimately, the outcomes are graphically scrutinized. The perspective solutions are cataloged and tabulated in detail. The present results are compared with published works in open literature and found a good agreement.

\section{Constitutive Relations}

While considering a non-Newtonian Eyring-Powell fluid model, the basic constituent relation is defined as [24]

$$
T=-p \mathbf{I}+\mathbf{Q}
$$

where

$$
\begin{aligned}
\mathbf{Q} & =\mu_{o} \mathbf{A}_{1}+\frac{1}{\beta^{*} \bar{\gamma}} \sinh ^{-1}\left(\frac{\bar{\gamma}}{C^{*}}\right) \mathbf{A}_{1}, \\
\mathbf{A}_{1} & =\operatorname{grad} \mathbf{V}+(\operatorname{grad} \mathbf{V})^{\mathrm{tr}}, \\
\bar{\gamma} & =\left[\frac{1}{2} \operatorname{tr}\left(\mathbf{A}_{1}\right)^{2}\right]^{(1 / 2)}, \\
\mathbf{V} & =[u(x, y), v(x, y), 0] .
\end{aligned}
$$

In abovementioned expressions, $T$ is Cauchy stress tensor, $p$ represents pressure, $\mathbf{I}$ represents identity tensor, $\mathbf{Q}$ is the extrastress tensor, $\mu$ describes dynamic viscosity, $\mathbf{A}_{1}$ is the first Rivilin-Ericksen tensor, $\bar{\gamma}$ is shear rate viscosity, $\beta^{*}$ and $C^{*}$ are the material constants, and $\mathrm{V}$ is the described velocity field. Now, up to second-order approximation of $\sinh ^{-1}$ function leads the expression towards

$$
\sinh ^{-1}\left(\frac{\bar{\gamma}}{C^{*}}\right) \approx\left(\frac{\bar{\gamma}}{C^{*}}-\frac{1}{6}\left(\frac{\bar{\gamma}}{C^{*}}\right)^{3}\right), \quad \text { with }\left|\bar{\gamma} / C^{*}\right| \ll 1 \text {. }
$$

After the simplification of boundary layer approximation, the flow equation for the Eyring-Powell fluid model now takes the form:

$$
u \frac{\partial u}{\partial x}+v \frac{\partial u}{\partial y}=v \frac{\partial^{2} u}{\partial y^{2}}+\frac{1}{\rho_{f} \beta^{*} C^{*}} \frac{\partial^{2} u}{\partial y^{2}}-\frac{1}{2 \rho_{f} \beta^{*} C^{* 3}}\left(\frac{\partial u}{\partial y}\right)^{2}\left(\frac{\partial^{2} u}{\partial y^{2}}\right)
$$

\section{Model Formulation}

The modeling is about a two-dimensional steady flow of incompressible non-Newtonian (Eyring-Powell) liquid within the influence of the applied magnetic field. The flow region is basically confined to $y>0$. To magnetize the liquid, a magnetic dipole having adequate intensity is placed over a stretching sheet in which $x$-axis lies horizontally to give rise a magnetic field whereas the $y$-axis is taken perpendicularly to the stretching surface. The region of flow is exposed by a uniform transverse magnetic field $=\left(0, B_{o}, 0\right)$ and a uniform electric field $=\left(0,0,-E_{o}\right)$ given, which are sufficiently applied across the stretching sheet. From Maxwell's equation, it is known that $\Delta \cdot \mathbf{B}=0$ and $\Delta \cdot \mathbf{E}=0$. So, the fluid is saturated with the magnetic field due to the presence of magnetic dipole. The stretching velocity is $u_{w}=a x$, and the perspective sheet is taken linearly stretchable to generate the flow, where " $a$ " is greater than zero and is the stretching rate, as shown in the schematic demonstration of the flow model in Figure 1. Further, for liquid magnetization, the magnetic dipole is positioned in such a way that its distance below $x$ axis is given by " $\mathrm{c}$ " and center on $y$-axis. The temperature at 


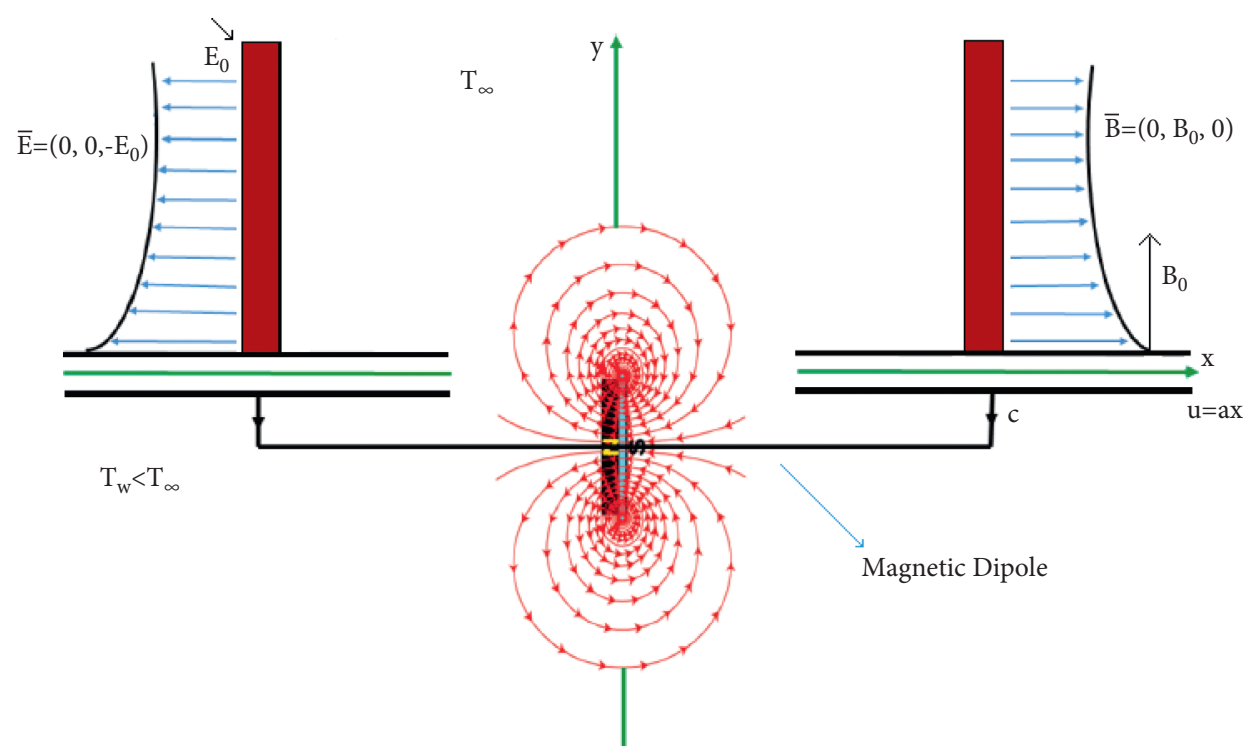

Figure 1: Schematic demonstration of flow model configuration.

the stretching surface is $T_{w}$. The Buongiorno model is accounted. The ramifications of activation energy and nonLinear thermal radiation are also taken into consideration.
For this flow model and in regard of aforesaid assumptions, the respective governing equations are $[16,17,24]$

$$
\begin{aligned}
& \frac{\partial u}{\partial x}+\frac{\partial v}{\partial y}=0 \\
& u \frac{\partial u}{\partial x}+v \frac{\partial u}{\partial y}=v \frac{\partial^{2} u}{\partial y^{2}}+\frac{1}{\rho_{f} \beta^{*} C^{*}} \frac{\partial^{2} u}{\partial y^{2}}-\frac{1}{2 \rho_{f} \beta^{*} C^{* 3}}\left(\frac{\partial u}{\partial y}\right)^{2}\left(\frac{\partial^{2} u}{\partial y^{2}}\right)+\frac{\lambda_{o} M}{\rho_{f}} \frac{\partial H}{\partial x}+\frac{\sigma}{\rho_{f}}\left(E_{o} B_{o}-B_{o}^{2} u\right) \\
& u \frac{\partial T}{\partial x}+v \frac{\partial T}{\partial y}+\frac{\lambda_{o}}{c_{p} \rho_{f}} T \frac{\partial M}{\partial T}\left(u \frac{\partial H}{\partial x}+v \frac{\partial H}{\partial y}\right)=\frac{k}{c_{p} \rho_{f}} \frac{\partial^{2} T}{\partial y^{2}}+\frac{16 \sigma^{*}}{3 c_{p} \rho_{f} \delta^{*}} \frac{\partial}{\partial y}\left(T^{3} \frac{\partial T}{\partial y}\right) \\
& +\tau\left[D_{B}\left(\frac{\partial C}{\partial y} \frac{\partial T}{\partial y}\right)+\frac{D_{T}}{T_{\infty}}\left(\frac{\partial T}{\partial y}\right)^{2}\right]+\frac{\sigma}{\rho_{f} c_{p}}\left(u B_{o}-E_{o}\right)^{2}+\frac{\mu}{c_{p} \rho_{f}}\left(\frac{\partial u}{\partial y}\right)^{2} \\
& u \frac{\partial C}{\partial x}+v \frac{\partial C}{\partial y}=D_{B} \frac{\partial^{2} C}{\partial y^{2}}+\frac{D_{T}}{T_{\infty}} \frac{\partial^{2} T}{\partial y^{2}}-k_{r}^{2}\left(C-C_{\infty}\right)\left(\frac{T}{T_{\infty}}\right)^{n} \exp \left(\frac{-E_{a}}{\kappa T}\right)
\end{aligned}
$$

where kinematic consistency is represented by $\nu=(\mu / \rho), \rho_{f}$ is the base liquid density, $\sigma$ is the representation of electrical conductivity, $H$ is the imposed magnetic field in the perpendicular direction along $y$-axis, $M$ represents the magnetization factor, fluid's temperature is given by $T$, specific heat capacity of the nanoparticle material is denoted by $c_{p}, C$ is the nanoparticle fluid concentration, $D_{B}$ and $D_{T}$ are the Brownian diffusion coefficient and thermophoretic diffusion coefficient, $E_{a}$ is denoted as the activation energy, $\beta^{*}$ and $C^{*}$ are the material constants, $\kappa$ is the Boltzmann constant, and $k_{r}^{2}$ is the chemical reaction rate constant. The surface coordinates both perpendicular and parallel are $y$ and $x$, and the velocity components in the direction of $x$ and $y$ are $u$ and $v$.
The correlated boundary conditions for the above PDEs have been taken as

$$
\begin{cases}u=a x, v=0, T=T_{w}, C=C_{w}, & \text { at } y=0, \\ u \longrightarrow 0, v \longrightarrow 0, T \longrightarrow T_{\infty}, C \longrightarrow C_{\infty}, & \text { at } y \longrightarrow \infty\end{cases}
$$

In boundary conditions, the stretching rate of the sheet is represented by " $a$, , $T_{w}$ denotes the hot fluid temperature, while $T_{\infty}$ and $C_{\infty}$ represent the ambient temperature and ambient concentration, respectively. It can be seen here that two forces constitute same magnitude but opposite orientations are employed to the sheet, while the origin is kept constant. The boundary condition $\left.u\right|_{y=0}=a x$ depicts the 
stretching velocity whereas $\left.v\right|_{y=0}=0$ demonstrates that the surface is impermeable, exhibiting no suction/injection. Further, $\left.u\right|_{y \rightarrow \infty} \longrightarrow 0$ physically describes that the freestream velocity is zero, while $\left.T\right|_{y \rightarrow \infty} \longrightarrow T_{\infty}$ signifies the ambient fluid temperature and $\left.C\right|_{y \longrightarrow \infty} \longrightarrow C_{\infty}$ reflects the ambient fluid concentration.

The liquid flow is influenced by the magnetic field that is subjected to the concept of magnetic dipole whose scalar potential is $\Phi^{*}$. Thus, the expression for the magnetic scalar potential, say $\Phi^{*}$, is expressed as

$$
\Phi^{*}=\frac{\gamma^{*}}{2 \pi}\left(\frac{x}{x^{2}+(y+c)^{2}}\right),
$$

where $\gamma^{*}$ basically points out the strength of magnetic dipole. For this flow, we considered that the field of magnetic force is conservative. We know that any conservative force can be expressed as a scalar of any potential function. So, in this case, the scalar potential function is represented by $\Phi^{*}$.

The components of the magnetic field along with its magnitude are given by $H_{x}$ and $H_{y}$ :

$$
\begin{aligned}
& H_{x}=-\frac{\partial \Phi^{*}}{\partial x}=\frac{\gamma^{*}}{2 \pi}\left[\frac{x^{2}-(y+c)^{2}}{\left(x^{2}+(y+c)^{2}\right)^{2}}\right] \\
& H_{y}=-\frac{\partial \Phi^{*}}{\partial y}=\frac{\gamma^{*}}{2 \pi}\left[\frac{2 x(y+c)}{\left(x^{2}+(y+c)^{2}\right)^{2}}\right], \\
& H=\left[\left(\frac{\partial \Phi^{*}}{\partial x}\right)^{2}+\left(\frac{\partial \Phi^{*}}{\partial y}\right)^{2}\right]^{(1 / 2)} .
\end{aligned}
$$

By expanding using Taylor's configuration for $x$ up to order 2, then from equation (13), one can get these two forms:

$$
\begin{aligned}
& \frac{\partial H}{\partial x}=-\frac{\gamma^{*}}{2 \pi}\left[\frac{2 x}{(y+c)^{4}}\right], \\
& \frac{\partial H}{\partial y}=\frac{\gamma^{*}}{2 \pi}\left[-\frac{2}{(y+c)^{3}}+\frac{4 x^{2}}{(y+c)^{5}}\right] .
\end{aligned}
$$

The variation of magnetization $(M)$ via temperature can be determined by the utilization of below expression, that is,

$$
M=K^{*}\left(T_{\infty}-T\right) .
$$

Now, the stream function that has been introduced and assumed to satisfy the continuity equation is given below. For the conversion of (6)-(8) into the dimensionless form, the following appropriate similarity transformation has been applied:

$$
\left\{\begin{array}{l}
\psi(\zeta, \eta)=\nu \zeta f(\eta), \\
\eta=\left(\frac{a}{\nu}\right)^{(1 / 2)} y \\
\zeta=\left(\frac{a}{v}\right)^{(1 / 2)} x \\
\theta(\eta)=\frac{T-T_{\infty}}{T_{w}-T_{\infty}} \\
T=T+\left(T_{w}-T_{\infty}\right) \theta(\eta), \\
\phi(\eta)=\frac{C-C_{\infty}}{C_{\infty}} \\
C=C_{\infty}+\left(C_{\infty}\right) \phi(\eta) \\
u=\frac{\partial \psi}{\partial y}=a x f^{\prime}(\eta) \\
\partial x
\end{array}\right.
$$

where $\psi$ depicts the stream function, the respective $(\eta, \zeta)$ represents the dimensionless parameters, and $\theta(\eta)$ and $\phi(\eta)$ are the expressions for thermal and concentration distribution along with the velocity components.

By using transformation given in (16), our above governing model (6)-(8) has now turned into a dimensionless system and exhibits the perfect ODEs that are

$$
\begin{aligned}
& f^{\prime \prime \prime}(1+\delta)-\delta \varepsilon f^{\prime \prime 2} f^{\prime \prime \prime}+f f^{\prime \prime}-f^{\prime 2}-\frac{2 \beta \theta}{(\eta+\alpha)^{4}}+(\mathrm{Ha})^{2}\left(E_{1}-f^{\prime}\right)=0 \\
& (1+N) \theta^{\prime \prime}+\operatorname{Pr}\left(f \theta^{\prime}+\phi^{\prime} \theta^{\prime} N_{b}+N_{t}\left(\theta^{\prime}\right)^{2}\right)-\operatorname{PrEc}\left(\theta-\varepsilon_{1}\right) \beta\left[\frac{2 f^{\prime}}{(\eta+\alpha)^{4}}+\frac{4 f}{(\eta+\alpha)^{5}}\right] \\
& \quad+N\left[\left(\theta_{w}-1\right)^{3}\left(\theta^{3} \theta^{\prime \prime}+3 \theta^{2}\left(\theta^{\prime}\right)^{2}\right)+3\left(\theta_{w}-1\right)^{2}\left(2 \theta\left(\theta^{\prime}\right)^{2}+\theta^{2} \theta^{\prime \prime}\right)+3\left(\theta_{w}-1\right)\left(\theta \theta^{\prime \prime}+\left(\theta^{\prime}\right)^{2}\right)\right] \\
& \quad+2 \lambda \beta\left(\theta-\varepsilon_{1}\right)\left(\frac{f}{(\eta+\alpha)^{3}}\right)+\operatorname{PrEc}(\mathrm{Ha})^{2}\left(f^{\prime}-E_{1}\right)^{2}+\operatorname{PrEc}\left(f^{\prime \prime}\right)^{2}=0,
\end{aligned}
$$




$$
\phi^{\prime \prime}+\frac{N_{t}}{N_{b}} \theta^{\prime \prime}+\operatorname{Sc} f \phi^{\prime}-(\mathrm{Sc}) \sigma\left(1+\delta_{1} \theta\right)^{n} \exp \left(\frac{-E}{1+\delta_{1} \theta}\right) \phi=0
$$

While transforming the conventional model into a frame of ODEs, following parameters are introduced during this transformation to have such formulation:

$$
\begin{aligned}
& \operatorname{Pr}=\frac{\mu c_{p}}{K}, \\
& \beta=\frac{\gamma^{*} \rho_{f} \lambda_{0}}{2 \pi \mu^{2}} K\left(T_{\infty}-T_{w}\right), \\
& N=\frac{16 \sigma^{*} T_{\infty}^{3}}{3 k \delta^{*}}, \\
& \varepsilon_{1}=\frac{T_{\infty}}{T_{\infty}-T_{w}}, \\
& \delta_{1}=\frac{T_{w}-T_{\infty}}{T_{\infty}}, \\
& \alpha=c\left(\frac{a \rho_{f}}{\mu}\right)^{(1 / 2)} \text {, } \\
& \lambda=\frac{a \mu^{2}}{\rho_{f} k\left(T_{\infty}-T_{w}\right)}, \\
& N_{t}=\frac{\tau D_{T}\left(T_{w}-T_{\infty}\right)}{T_{\infty} \nu}, \\
& N_{b}=\frac{\tau D_{B} C_{\infty}}{v}, \\
& \theta_{w}=\frac{T_{w}}{T_{\infty}}, \\
& \mathrm{Sc}=\frac{v}{D_{B}}, \\
& \sigma=\frac{k_{r}^{2}}{a}, \\
& E=\frac{E_{a}}{\kappa T_{\infty}}, \\
& \varepsilon=\frac{a^{3} x^{2}}{2 C^{* 2} \nu}, \\
& \delta=\frac{1}{\mu \beta^{*} C^{*}}, \\
& \mathrm{Ha}^{2}=\frac{\sigma B_{0}}{\rho a}, \\
& E_{1}=\frac{E_{o}}{B_{o} a x},
\end{aligned}
$$

where prime $\left({ }^{\prime}\right)$ denotes the differentiation with respect to $\eta$ and the abovementioned parameter represents the Prandtl number, ferromagnetic interaction parameter, thermal radiation variable, temperature difference variable, dimensionless difference parameter, viscous dissipation parameter, thermophoretic parameter, Brownian motion parameter, temperature ratio variable, Schmidt number, chemical reaction rate constant, activation energy, fluid parameters $(\varepsilon, \delta)$, Hartmann number, and local electric parameter, respectively.

And, the boundary conditions at $(\eta=0$ and $\eta \longrightarrow \infty)$ regarding the ODE's (6)-(8) get the form:

$$
\left\{\begin{array}{l}
f^{\prime}(0)=1, \\
f(0)=0, \\
\theta(0)=1, \\
\phi(0)=1, \\
f^{\prime}(\infty) \longrightarrow 0, \\
\theta(\infty) \longrightarrow 0, \\
\phi(\infty) \longrightarrow 0 .
\end{array}\right.
$$

\section{Physical Quantities (Velocity, Thermal Gradient, and Solutal Gradient)}

In practical interest, the most significant physical quantities are $C_{f x}, \mathrm{Nu}_{x}$, and $\mathrm{Sh}_{x}$ which denote the coefficient of local skin friction, coefficient of local rate of heat transfer, and mass transfer rate coefficient, respectively. The mathematical formulation of the surface drag coefficient $\left(C_{f x}\right)$, thermal gradient $\left(\mathrm{Nu}_{x}\right)$, and solutal gradient $\left(\mathrm{Sh}_{x}\right)$ is defined as

$$
\begin{aligned}
C_{f x} & =\frac{\tau_{w}}{\rho_{f} a^{2} x^{2}}, \\
\mathrm{Nu}_{x} & =\frac{x q_{w}}{k\left(T_{w}-T_{\infty}\right)}, \\
\mathrm{Sh}_{x} & =\frac{x q_{m}}{-D_{B}\left(C_{\infty}\right)},
\end{aligned}
$$

where $\tau_{w}, q_{w}$, and $q_{m}$ are the representation of wall shear stress, heat flux, and mass flux, respectively, at the wall, i.e., at $y=0$, and are described as

$$
\begin{aligned}
& \tau_{w}=\left.\left(\mu+\frac{1}{\beta^{*} C^{*}}\right) \frac{\partial u}{\partial y}\right|_{y=0}-\left.\frac{1}{6 \beta^{*} C^{* 3}}\left(\frac{\partial u}{\partial y}\right)^{3}\right|_{y=0}, \\
& q_{w}=-\left.k \frac{\partial T}{\partial y}\right|_{y=0}-\left.\frac{16 \sigma^{*}}{3 \delta^{*}}\left(T^{3} \frac{\partial T}{\partial y}\right)\right|_{y=0}, \\
& q_{m}=-\left.D_{B} \frac{\partial C}{\partial y}\right|_{y=0} .
\end{aligned}
$$

The nondimensionalization of the surface drag coefficient which is also the velocity gradient $C_{f x}$, thermal gradient $\mathrm{Nu}_{x}$, and solutal gradient $\mathrm{Sh}_{x}$ using the similarity transformations is given as 


$$
\begin{aligned}
& \Rightarrow C_{f x}\left(\operatorname{Re}_{x}\right)^{(1 / 2)}=(1+\delta) f^{\prime \prime}(0)-\frac{\delta \varepsilon}{3}\left(f^{\prime \prime}(0)\right)^{3} \\
& \Rightarrow\left(\mathrm{Nu}_{x}\right) \operatorname{Re}_{x}^{-(1 / 2)}=-\theta^{\prime}(0)\left[1+N\left(1+\left(\theta_{w}-1\right) \theta(0)\right)^{3}\right] \\
& \Rightarrow\left(\mathrm{Sh}_{x}\right) \operatorname{Re}_{x}^{-(1 / 2)}=\phi^{\prime}(0),
\end{aligned}
$$

where $f^{\prime \prime}(0)$ depicts the nondimensional wall shear parameter, $\theta^{\prime}(0)$ represents the nondimensional rate of heat transfer at sheet, and $\phi^{\prime}(0)$ depicts nondimensionalized rate of mass transfer where the expression $\operatorname{Re}_{x}=\left(a x^{2} / \nu\right)$ is for local Reynolds number. It is more obvious that the field of flow is primarily affected by the parameter of ferromagnetic interaction $(\beta)$.

\section{Numerical Scheme}

The above highly nonlinear scheme is transformed to the boundary value problem (BVP) settled in equations (17)-(19) in the companionship of pertinent boundary conditions given in (21). The resulting system is scrutinized on numerical basis by the implementation of the $b v p 4 c$ scheme using computational software MATLAB.

Prior to initiate the numerical simulation, firstly we remould the higher order BVP into first order (IVP). Such reformation of BVP into IVP is localized on the basis of some restricted values as $(\eta \longrightarrow \infty)$ for a restricted domain boundary condition say $\left(\eta_{\infty}=5\right)$. For this, one can introduce the new settlement of dependent specified variables as follows:

$$
\begin{aligned}
f & =Y_{1}, \\
f^{\prime} & =Y_{2}, \\
f^{\prime \prime} & =Y_{3}, \\
f^{\prime \prime \prime} & =Y_{3}^{\prime}, \\
\theta & =Y_{4}, \\
\theta^{\prime} & =Y_{5}, \\
\theta^{\prime \prime} & =Y_{5}^{\prime}, \\
\phi & =Y_{6}, \\
\phi^{\prime} & =Y_{7}, \\
\phi^{\prime \prime}= & Y_{7}^{\prime} \Rightarrow Y_{3}^{\prime}=\frac{1}{A_{1}}\left[-Y_{1} Y_{3}+\left(Y_{2}\right)^{2}\right. \\
& \left.+\frac{2 \beta Y_{4}}{(\eta+\alpha)^{4}}-\mathrm{Ha}^{2}\left(E_{1}-Y_{2}\right)\right],
\end{aligned}
$$

where $A_{1}=\left[(1+\delta)-\delta \varepsilon\left(Y_{3}\right)^{2}\right]$, and

$$
\begin{aligned}
\Rightarrow Y_{5}^{\prime}= & \frac{1}{A_{2}}\left[-\operatorname{Pr}\left(Y_{1} Y_{5}+N_{b} Y_{7} Y_{5}+N_{t}\left(Y_{5}\right)^{2}\right)-3 N\left(\theta_{w}-1\right)\left(Y_{5}\right)^{2}\left[\left(Y_{4}\right)^{2}\left(\theta_{w}-1\right)^{2}+2 Y_{4}\left(\theta_{w}-1\right)+1\right]\right. \\
& +\operatorname{Pr} E_{c} \beta\left(Y_{4}-\varepsilon_{1}\right)\left[\frac{2 Y_{2}}{(\eta+\alpha)^{4}}+\frac{4 Y_{1}}{(\eta+\alpha)^{5}}\right]-2 \lambda \beta\left(Y_{4}-\varepsilon_{1}\right) \frac{Y_{1}}{(\eta+\alpha)^{3}}-\operatorname{Pr}_{c} \mathrm{Ha}^{2}\left(Y_{2}-E_{1}\right)^{2}-\operatorname{Pr} E_{c}\left(Y_{3}\right)^{2}
\end{aligned}
$$

where $A_{2}=\left[1+N\left(1+Y_{4}\left(\theta_{w}-1\right)\right)^{3}\right]$, and

$$
\Rightarrow Y_{7}^{\prime}=-\frac{N_{t}}{N_{b}} Y_{5}^{\prime}-\operatorname{Sc} Y_{1} Y_{7}+\operatorname{Sc} \sigma\left(1+\delta_{1} Y_{4}\right)^{n} \exp \left(\frac{-E}{1+\delta_{1} Y_{4}}\right) Y_{6},
$$

along with the boundary conditions:

$$
\left\{\begin{array}{l}
Y_{1}(0)=0, \\
Y_{2}(0)=1, \\
Y_{4}(0)=1, \\
Y_{6}(0)=1, \\
Y_{2}(\infty) \longrightarrow 0, \\
Y_{4}(\infty) \longrightarrow 0, \\
Y_{6}(\infty) \longrightarrow 0 .
\end{array}\right.
$$

The abovementioned IVP will be scrutinized using the numerical approach by the $b v p 4 c$ method on MATLAB. In order to attain the approximate solution, the domain of the observed problem has been taken within the interval as $\left[0, \eta_{\infty}\right]$, where $\eta_{\infty}$ defines a pertinent finite positive number of real line, and we take it as 5 during our implementation. Moreover, mesh resolution is created in grid continuation and tolerance of relative error in this method which is particularized as $10^{-7}$.

\section{Results and Discussion}

In this article, the main focus of this subsequent discussion is to tackle the physical and graphical features. The miscellaneous parameters in the respective field of flow are taken into account. The equations involved in this flow are a highly nonlinear coupled set of ODEs experiencing the boundary conditions as an outcome of transformation of PDEs. The system of third-order ODEs is then interpreted by means of the $b v p 4 c$ scheme using MATLAB with given comparison using MAPLE dsolve. Then, the ramifications under the varying physical parameters with pertinent values of ferrohydrodynamic interaction $(\beta)$, Prandtl number (Pr), Brownian motion 
parameter $(\mathrm{Nb})$, thermophoresis parameter $(\mathrm{Nt})$, electric parameter $E_{1}$, Schmidt number (Sc), temperature ratio variable $\left(\theta_{w}\right)$, thermal radiation parameter $(N)$, activation energy $(E)$, Eckert number $(\mathrm{Ec})$, chemical reaction rate constant $(\sigma)$, temperature difference parameter $\left(\delta_{1}\right)$, Sherwood number (Sh) against the axial velocity $f^{\prime}(\eta)$, thermal field $\theta(\eta)$, and the concentration profile $\phi(\eta)$ are explored. The present research work is numerically computed and tabulated for velocity, thermal, and solutal gradient and then graphically scrutinized in detail. A parallel comparison is given for both the methods to demonstrate that bvp $4 \mathbf{c}$ casts a less computational cost and gives more accuracy of numerical computation. This method is an effective solver, with an approach towards the increasing convergence criteria. The boundary conditions of the BVP (boundary value problem) and the algorithm that is handled in bvp4c typically make it a direct method because the final equation to solve on the defined domain of interval is algebraic. On the contrary, ds olve take less time to execute and casts less computational cost. In ds olve MAPLE, both initial and BVP can be numerically computed. For a system input, the type of problem is automatically recognized. As both are numerical methods, the numerical method always works with iteration. Such methods are easy to compute. When the analytical solution does not exist to solve a system, then numerical solution is preferred. This section includes the physical interpretation of physical parameters that cast impact on the flow field by variation. The outcomes of this research work have found a good agreement with the prior published works which indicates the sustainability of the abovementioned research studies.

Table 1 highlights the comparative outcomes of velocity gradient $C_{f}\left(\operatorname{Re}_{x}\right)^{(1 / 2)}$ along with the analysis given in $[13,24]$, showing a good agreement with prior research studies. Table 2 shows the effects of $C_{f}\left(\operatorname{Re}_{x}\right)^{(1 / 2)}$ for different values of $\varepsilon, \delta, \mathrm{Ha}, E 1$, and $\beta$. It was observed that rising values of $\delta$, Ha, and $\beta$ decrease the skin friction coefficient. Besides this, for larger $\varepsilon$ and $E 1$ parameters, $C_{f}\left(\mathrm{Re}_{x}\right)^{(1 / 2)}$ reveals an increment trend, whereas in Table 3 , the outcomes elaborate the parametric effects of $\mathrm{Pr}, \mathrm{N}, \theta_{w}, \mathrm{Ec}, \mathrm{Ha}, \mathrm{Nb}$, and $\mathrm{Nt}$ against the thermal gradient $\mathrm{Nu}\left(\mathrm{Re}_{x}\right)^{-(1 / 2)}$. Here, larger estimation in $\operatorname{Pr}, \mathrm{N}$, and $\theta_{w}$ parameters yields the ascending profile for $\mathrm{Nu}\left(\mathrm{Re}_{x}\right)^{-(1 / 2)}$, while for higher values of $\mathrm{Ec}, \mathrm{Ha}, \mathrm{Nb}$, and $\mathrm{Nt}$ parameters, $\mathrm{Nu}\left(\mathrm{Re}_{x}\right)^{-(1 / 2)}$ reveals a descending trend. Table 4 is scrutinized about the $-\mathrm{Sh}\left(\operatorname{Re}_{x}\right)^{-(1 / 2)}$ outcomes for varying values of $\sigma, \delta_{1}, \mathrm{Sc}$, and $\mathrm{Nb}$, and the results evaluated that, for increase in these parameters, the Sherwood number rises, while for higher $E, \mathrm{Ha}, \beta$, and Nt parameters, the solutal gradient falls down gradually.

So far, the respective graphical approach is considered, and Figure 2 depicts the graphical scrutinization of velocity profile versus $\eta$ for different values of local electric field parameter $E_{1}$. It is observed that the velocity profile exhibits an increase in the boundary layer thickness with uplifting values of electric parameter $E_{1}$ but more significantly, a little bit away from the stretching sheet. The overall analysis highlighted the theme that the impact cast by $E_{1}$ is basically to shift the streamlines little away from the stretchable boundary because the Lorentz force rises due to effect of the electric field and acts as an accelerating force in decreasing the frictional resistance effects.

Figure 3 illustrates the impact of $\beta$ against the velocity profile $f^{\prime}(\eta)$. The decaying trend emerging via larger $\beta$ implies that the fluid motion is the decreasing function of the $\beta$ factor. In physical simulation, an uplift in the values of $\beta$ causes to enhance the resistive force (called the Lorentz force) in fluid flow that slows down the motion of fluid, as the viscosity becomes more concentrated for larger values of $\beta$ that ultimately declines the velocity profile. As expected, $f^{\prime}(\eta)$ diminishes via larger $\beta$.

In Figure 4, the Hartmann number $(\mathrm{Ha})$ is now to indicated a decreasing behaviour for the velocity profile in the boundary layer that comes up with the effect in thinning the boundary layer thickness.

Figure 5 indicates the effects of $(\mathrm{Ha})$ on the thermal profile. Temperature distribution increases with continual rise in $(\mathrm{Ha})$ number. The figure gives the result that the uniform transverse magnetic field participates in thickening the thermal boundary layer. This concept comes from the fact that the applied uniform transverse magnetic field gives rise to a body force such as Lorentz force which causes an opposing reaction in the motion, thereby enhancing the temperature profile.

Figure 6 demonstrates the impact of increasing values of $E_{1}$ parameter against the temperature profile. As with the continuous rising in $E_{1}$ parametric values, the thermal graph shows a decline behaviour.

Figure 7 proclaims the stimulus of ferrohydrodynamic interaction variable $(\beta)$ on $\theta(\eta)$. One can observe that, with an intensification of $\beta, \theta(\eta)$ is an enhancing function of $\beta$. Through larger $\beta$ approximations, the resistive force upsurges due to which the fluid movement faces high resistance during its flow. As a result, this friction converts the valuable mechanical energy into heat energy that augments the temperature. Hence, $\theta(\eta)$ rises for larger $\beta$ which is evident in Figure 3.

Now, Figure 8 poses to display the behaviour of $\theta(\eta)$ due to discrete values of $(\mathrm{Pr})$. The increasing trend of $(\mathrm{Pr})$ delineates the thermal graph. In the physical sense, since the Prandtl Number is dimensionless and defines as a relation (ratio) of molecular diffusivity over thermal diffusivity, so (Pr) and thermal diffusivity are inversely inter-related, noticing the reduction in the proficiency of energy transportation. Also, the thickness of the boundary layer compressed just about to wall. The increment in (Pr) parameter drops the thermal diffusivity and eventually makes the fluids temperature to fall down.

Curves of $\theta(\eta)$ examined through Brownian motion parameter $(\mathrm{Nb})$ for absolute variation are elaborated in Figure 9. The graph scrutinizes that increase in $(\mathrm{Nb})$ typically enhances the temperature profile. The description is quite reliable. As the $(\mathrm{Nb})$ parameter is in accordance with the concentration gradient when giving the variation to this parameter results in boosting the thermal energy of fluid, it ultimately brings the temperature profile up. It can also be observed that, for larger $(\mathrm{Nb})$, the collision between the 
TABle 1: Comparative outcomes of $C_{f}\left(\operatorname{Re}_{x}\right)^{(1 / 2)}$ with Ref. [13] and Ref. [24] when $\beta=0$.

\begin{tabular}{lccccccccccccc}
\hline$\varepsilon$ & $\delta$ & $f^{\prime \prime}(0)$ & {$[13]$} & {$[24]$} & MATLAB & MAPLE & $\varepsilon$ & $\delta$ & $f^{\prime \prime}(0)$ & {$[13]$} & [24] & MATLAB & MAPLE \\
\hline 0.0 & 0.2 & -0.9129 & -1.0954 & -1.0954 & -1.0955 & -1.0955 & 0.0 & 0.4 & -0.8452 & -1.1832 & -1.1832 & -1.1833 & -1.1833 \\
0.1 & 0.2 & -0.9159 & -1.0940 & -1.0940 & -1.0940 & -1.0940 & 0.1 & 0.4 & -0.8494 & -1.1808 & -1.1809 & -1.1810 & -1.1809 \\
0.2 & 0.2 & -0.9190 & -1.0924 & -1.0925 & -1.0925 & -1.0924 & 0.2 & 0.4 & -0.8537 & -1.1784 & -1.1784 & -1.1786 & -1.1785 \\
0.3 & 0.2 & -0.9222 & -1.0909 & -1.0909 & -1.0910 & -1.0910 & 0.3 & 0.4 & -0.8581 & -1.1776 & -1.1760 & -1.1761 & -1.1761 \\
0.4 & 0.2 & -0.9255 & -1.0894 & -1.0894 & -1.0895 & -1.0895 & 0.4 & 0.4 & -0.8627 & -1.1735 & -1.1735 & -1.1735 & -1.1736 \\
0.5 & 0.2 & -0.9288 & -1.0878 & -1.0878 & -1.0879 & -1.0879 & 0.5 & 0.4 & -0.8676 & -1.1710 & -1.1710 & -1.1711 & -1.1711 \\
0.6 & 0.2 & -0.9340 & -1.0862 & -1.0863 & -1.0864 & -1.0863 & 0.6 & 0.4 & -0.8752 & -1.1684 & -1.1684 & -1.1685 & -1.1684 \\
0.7 & 0.2 & -0.9358 & -1.0847 & -1.0847 & -1.0847 & -1.0847 & 0.7 & 0.4 & -0.8778 & -1.1658 & -1.1658 & -1.1658 & -1.1659 \\
0.8 & 0.2 & -0.9394 & -1.0830 & -1.0830 & -1.0831 & -1.0831 & 0.8 & 0.4 & -0.8834 & -1.1631 & -1.1631 & -1.1632 & -1.1632 \\
0.9 & 0.2 & -0.9432 & -1.0814 & -1.0814 & -1.0815 & -1.0815 & 0.9 & 0.4 & -0.8891 & -1.1603 & -1.1603 & -1.1604 & -1.1604 \\
1.0 & 0.2 & -0.9470 & -1.0798 & -1.0798 & -1.0798 & -1.0798 & 1.0 & 0.4 & -0.8952 & -1.1576 & -1.1576 & -1.1576 & -1.1576 \\
\hline
\end{tabular}

TABLE 2: Calculation of velocity gradient $C_{f}\left(\operatorname{Re}_{x}\right)^{(1 / 2)}$ for different variations of $\varepsilon, \delta$, Ha, $E 1$, and $\beta$.

\begin{tabular}{|c|c|c|c|c|c|c|}
\hline$\varepsilon$ & $\delta$ & $\mathrm{Ha}$ & $E 1$ & $\beta$ & MATLAB & MAPLE \\
\hline 0.1 & & & & & -1.87887 & -1.87887 \\
\hline 0.3 & & & & & -1.87735 & -1.87734 \\
\hline 0.5 & & & & & -1.87581 & -1.87581 \\
\hline \multirow[t]{17}{*}{0.7} & & & & & -1.87424 & -1.87425 \\
\hline & 0.01 & & & & -1.86916 & -1.86916 \\
\hline & 0.02 & & & & -1.87346 & -1.87346 \\
\hline & 0.03 & & & & -1.87776 & -1.87776 \\
\hline & 0.04 & & & & -1.88205 & -1.88205 \\
\hline & & 0.0 & & & -1.48350 & -1.48350 \\
\hline & & 0.5 & & & -1.59485 & -1.59485 \\
\hline & & 1.0 & & & -1.87346 & -1.87346 \\
\hline & & 1.5 & & & -2.23559 & -2.23559 \\
\hline & & & 0.0 & & -1.88238 & -1.88238 \\
\hline & & & 0.5 & & -1.47368 & -1.47368 \\
\hline & & & 1.0 & & -1.10812 & -1.10812 \\
\hline & & & 1.5 & & -0.766750 & -0.766750 \\
\hline & & & & 0.2 & -1.56758 & -1.56758 \\
\hline & & & & 0.8 & -2.02786 & -2.02786 \\
\hline & & & & 1.4 & -2.49824 & -2.49824 \\
\hline & & & & 2.0 & -2.98272 & -2.98272 \\
\hline
\end{tabular}

nano-sized particles is augmented in flow that yields convection due to which the temperature enhances in a definite sense.

Figure 10 is plotted to display the ramifications of thermophoresis parameter $(\mathrm{Nt})$ over $\theta(\eta)$ for discrete values. The graph illustrates that rising the $(\mathrm{Nt})$ parameter intensifies the temperature field in the zone of boundary layer aligned (horizontal) to the wall. In fact, the (Nt) parameter is correlated with the temperature gradient. This parameter tends to transport the fluid particles with its positive variation from a region of higher energy level to the colder one and intensifies the fluid temperature in the flow regime. One can say that, while uplifting its parametric values, it increases the thermal energy responsible for augmentation in the temperature field.

Figure 11 is designed to demonstrate the impacts of temperature ratio variable $\left(\theta_{w}\right)$ against $\theta(\eta)$. Here, thermal layer and $\theta(\eta)$ are in correspondence as well as $\theta_{w}$ upsurges. It physically elaborates that the outcome of uplift in this parameter rises the surface temperature $T_{w}$ and ultimately
TABLE 3: Calculation of thermal gradient $\mathrm{Nu}\left(\operatorname{Re}_{x}\right)^{-(1 / 2)}$ for different variations of $\mathrm{Pr}, N, \theta_{w}$, Ec, $\mathrm{Ha}, \mathrm{Nb}$, and $\mathrm{Nt}$.

\begin{tabular}{|c|c|c|c|c|c|c|c|c|}
\hline $\operatorname{Pr}$ & $N$ & $\theta_{w}$ & Ec & $\mathrm{Ha}$ & $\mathrm{Nb}$ & $\mathrm{Nt}$ & MATLAB & MAPLE \\
\hline 1.4 & & & & & & & 0.44767 & 0.44767 \\
\hline 1.6 & & & & & & & 0.46885 & 0.46885 \\
\hline 1.8 & & & & & & & 0.48721 & 0.48721 \\
\hline 2.0 & & & & & & & 0.50294 & 0.50294 \\
\hline & 0.2 & & & & & & 0.50294 & 0.50294 \\
\hline & 0.8 & & & & & & 0.52715 & 0.52715 \\
\hline & 1.2 & & & & & & 0.54414 & 0.54413 \\
\hline & 1.6 & & & & & & 0.56170 & 0.56171 \\
\hline & & 0.5 & & & & & 0.50939 & 0.50939 \\
\hline & & 1.2 & & & & & 0.57314 & 0.57314 \\
\hline & & 1.5 & & & & & 0.62345 & 0.62345 \\
\hline & & 1.8 & & & & & 0.68393 & 0.68393 \\
\hline & & & 0.0 & & & & 0.52168 & 0.52168 \\
\hline & & & 0.05 & & & & 0.42819 & 0.42819 \\
\hline & & & 0.1 & & & & 0.33519 & 0.33519 \\
\hline & & & 0.15 & & & & 0.24266 & 0.24266 \\
\hline & & & & 0.0 & & & 0.56934 & 0.56934 \\
\hline & & & & 0.5 & & & 0.54992 & 0.54992 \\
\hline & & & & 1.0 & & & 0.50294 & 0.50294 \\
\hline & & & & 1.5 & & & 0.44744 & 0.44744 \\
\hline & & & & & 0.1 & & 0.58396 & 0.58396 \\
\hline & & & & & 0.3 & & 0.43159 & 0.43159 \\
\hline & & & & & 0.5 & & 0.31452 & 0.31452 \\
\hline & & & & & 0.7 & & 0.22617 & 0.22617 \\
\hline & & & & & & 0.1 & 0.54461 & 0.54461 \\
\hline & & & & & & 0.3 & 0.46523 & 0.46523 \\
\hline & & & & & & 0.5 & 0.40012 & 0.40012 \\
\hline & & & & & & 0.7 & 0.34647 & 0.34647 \\
\hline
\end{tabular}

leads to more heat transportation. Therefore, rise in $\theta_{w}$ results in intensification of the temperature field.

Curves that occur in Figure 12 are designed to interpret the role of the thermal radiation parameter $(N)$ versus $\theta(\eta)$. This figure reveals that, for higher estimation of $(N)$ parameter, it escalates the thermal profile. The fact is the mean absorption coefficient $\left(\delta^{*}\right)$ that tends to decay as $(N)$ parameter accelerates. As a result, the diffusion flux comes into being, observing that the radiation parameter has a momentous supremacy over this profile.

Figure 13 highlights the characteristics of the Eckert Number (Ec) against $\theta(\eta)$. The major aspect of this graphical approach is to signify that the thermal layer 
TABLE 4: Calculation of solutal gradient $-\mathrm{Sh}\left(\operatorname{Re}_{x}\right)^{-(1 / 2)}$ for different variations of $H, \beta, \sigma, \delta_{1}, E, \mathrm{Sc}, \mathrm{Nt}$, and $\mathrm{Nb}$.

\begin{tabular}{|c|c|c|c|c|c|c|c|c|c|}
\hline $\mathrm{Ha}$ & $\beta$ & $\sigma$ & $\delta_{1}$ & $E$ & $\mathrm{Sc}$ & $\mathrm{Nt}$ & $\mathrm{Nb}$ & MATLAB & MAPLE \\
\hline 0.0 & & & & & & & & 1.50837 & 1.50837 \\
\hline 0.5 & & & & & & & & 1.47915 & 1.47915 \\
\hline 1.0 & & & & & & & & 1.40832 & 1.40832 \\
\hline 1.5 & & & & & & & & 1.32393 & 1.32393 \\
\hline & 0.2 & & & & & & & 1.45203 & 1.45203 \\
\hline & 0.8 & & & & & & & 1.38512 & 1.38512 \\
\hline & 1.4 & & & & & & & 1.30851 & 1.30851 \\
\hline & 2.0 & & & & & & & 1.21669 & 1.21669 \\
\hline & & 1.0 & & & & & & 1.42977 & 1.42977 \\
\hline & & 2.0 & & & & & & 1.46009 & 1.46009 \\
\hline & & 3.0 & & & & & & 1.49002 & 1.49002 \\
\hline & & 4.0 & & & & & & 1.51957 & 1.51957 \\
\hline & & & 1.0 & & & & & 1.43594 & 1.43594 \\
\hline & & & 2.0 & & & & & 1.52360 & 1.52360 \\
\hline & & & 3.0 & & & & & 1.64272 & 1.64272 \\
\hline & & & 4.0 & & & & & 1.77391 & 1.77391 \\
\hline & & & & 4.5 & & & & 1.42786 & 1.42786 \\
\hline & & & & 5.0 & & & & 1.41879 & 1.41879 \\
\hline & & & & 5.5 & & & & 1.41258 & 1.41258 \\
\hline & & & & 6.0 & & & & 1.40832 & 1.40832 \\
\hline & & & & & 4.5 & & & 1.11125 & 1.11125 \\
\hline & & & & & 5.0 & & & 1.21590 & 1.21590 \\
\hline & & & & & 5.5 & & & 1.31464 & 1.31464 \\
\hline & & & & & 6.0 & & & 1.40832 & 1.40832 \\
\hline & & & & & & 0.1 & & 1.49178 & 1.49178 \\
\hline & & & & & & 0.3 & & 1.34616 & 1.34616 \\
\hline & & & & & & 0.5 & & 1.27103 & 1.27103 \\
\hline & & & & & & 0.7 & & 1.24322 & 1.24322 \\
\hline & & & & & & & 0.1 & 1.09703 & 1.09703 \\
\hline & & & & & & & 0.3 & 1.50581 & 1.50581 \\
\hline & & & & & & & 0.5 & 1.57398 & 1.57398 \\
\hline & & & & & & & 0.7 & 1.59549 & 1.59549 \\
\hline
\end{tabular}

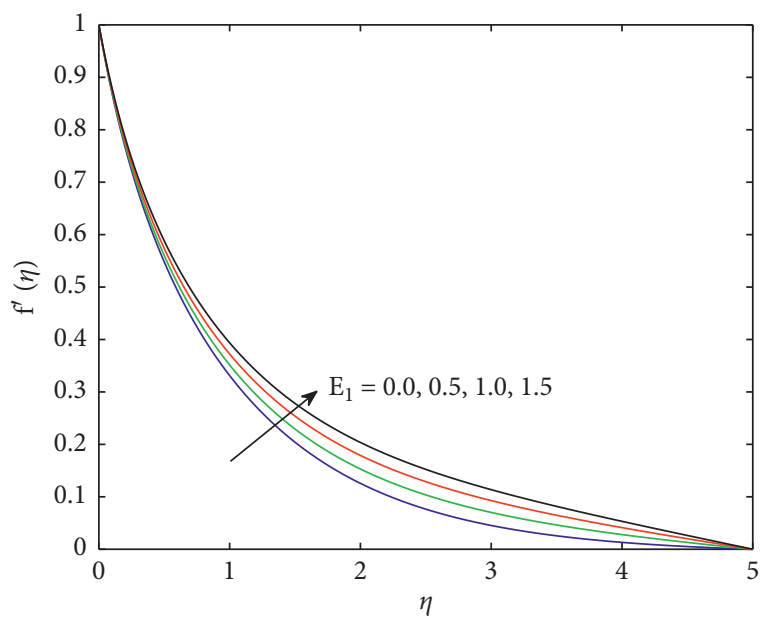

FIgURE 2: Velocity profile versus $E_{1}$.

intercorrelated with $\theta(\eta)$; both depict the ascendancy for larger variation in the $(\mathrm{Ec})$ profile. Due to rise in $(\mathrm{Ec})$, the thermal energy in fluid is accumulated that leads to friction force that inflated this thermal graph.

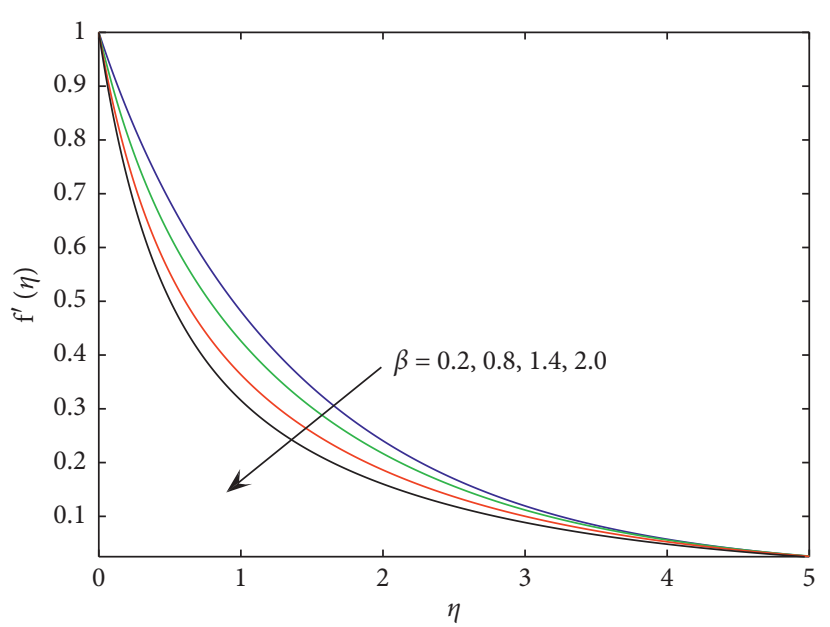

FIGURE 3: Velocity profile versus $\beta$.

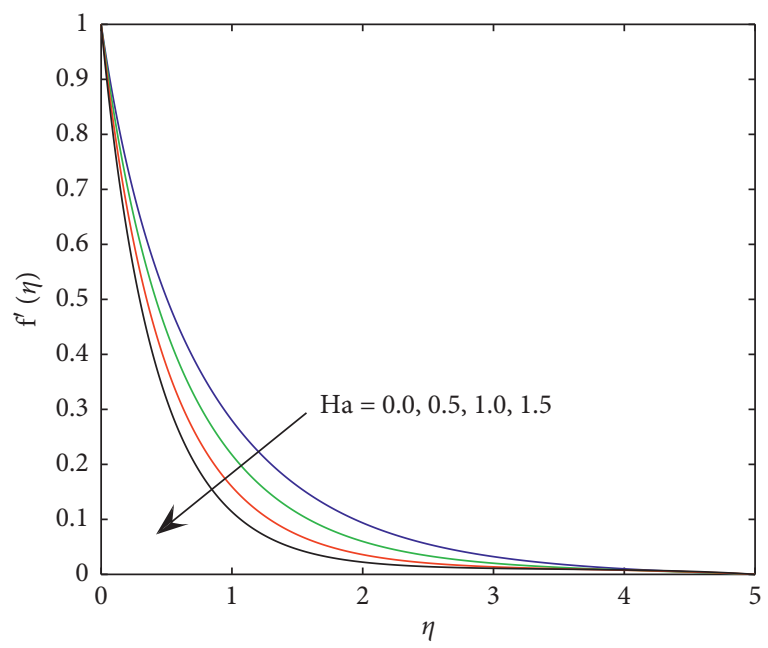

FIgURe 4: Velocity profile versus Ha.

Finally, to observe the consequences against the concentration profile $\phi(\eta)$, the sundry parameters such as $(\mathrm{Nb})$, $(\mathrm{Nt}),(\mathrm{Sc}),(E),\left(\delta_{1}\right),(\sigma), \mathrm{Ha}$, and $\beta$ involve discrete variation and cast impact on $\phi(\eta)$. These repercussions can be observed with the help of following description.

In Figure 14, the graphical scheme exhibits the effect of the Brownian motion parameter $(\mathrm{Nb})$ against $\phi(\eta)$. It can be seen that, for higher extension in $(\mathrm{Nb})$ values, the associated thermal layer along with the $\phi(\eta)$ profile deteriorates sharply. As, for higher $\mathrm{Nb}$, collision among nanoparticles rises that tends to move them in irregular manner, which results in diminishing the corresponding solutal layer.

Figure 15 describes the characteristics of the concentration field affected via the thermophoresis parameter $(\mathrm{Nt})$. Significantly, the fluid's thermal conductivity reinforces because of the existence of nanoparticles for greater $(\mathrm{Nt})$.

Curves subjected to Figure 16 explore the impacts of the Schmidt Number $(\mathrm{Sc})$ over $\phi(\eta)$. The positive increment in (Sc) profile causes permanent diminution the concentration field. Briefly, Sc and momentum diffusivity factor $\left(D_{B}\right)$ are 


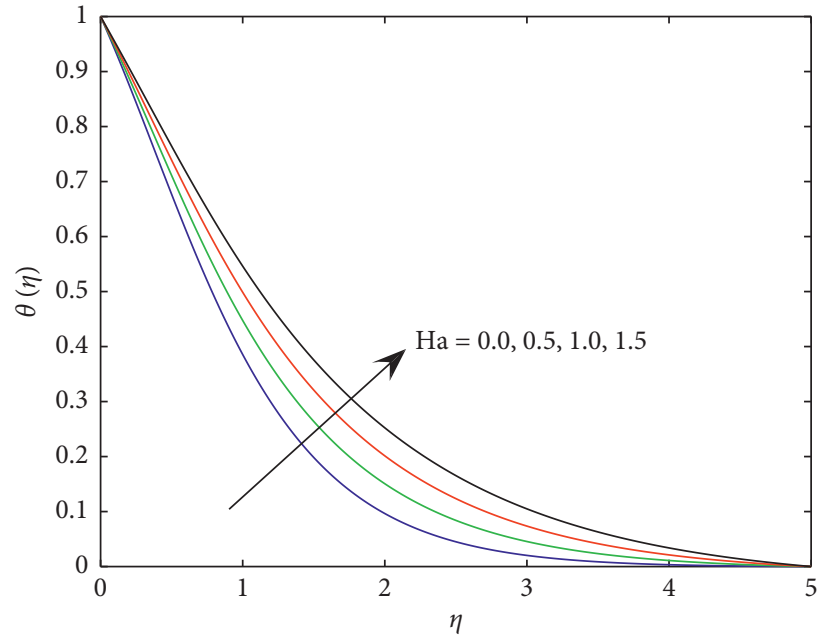

FIgURe 5: Temperature profile versus Ha.

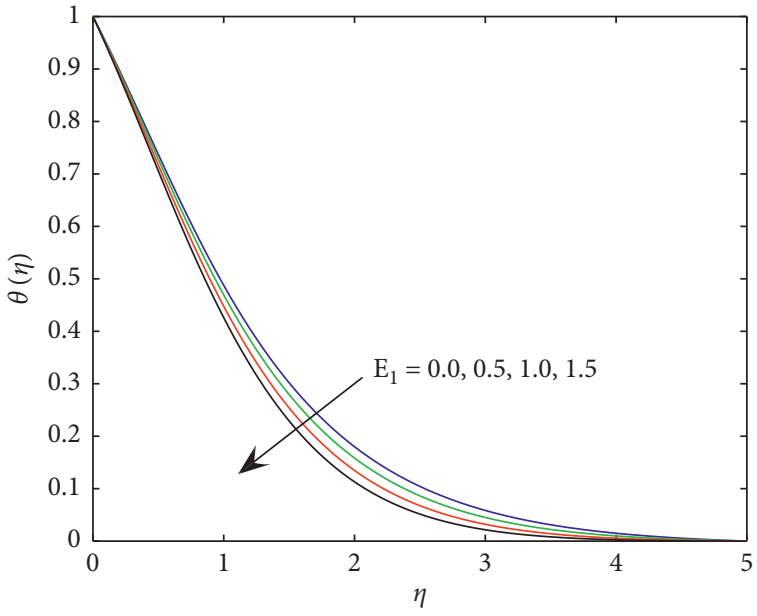

Figure 6: Temperature profile versus $E_{1}$.

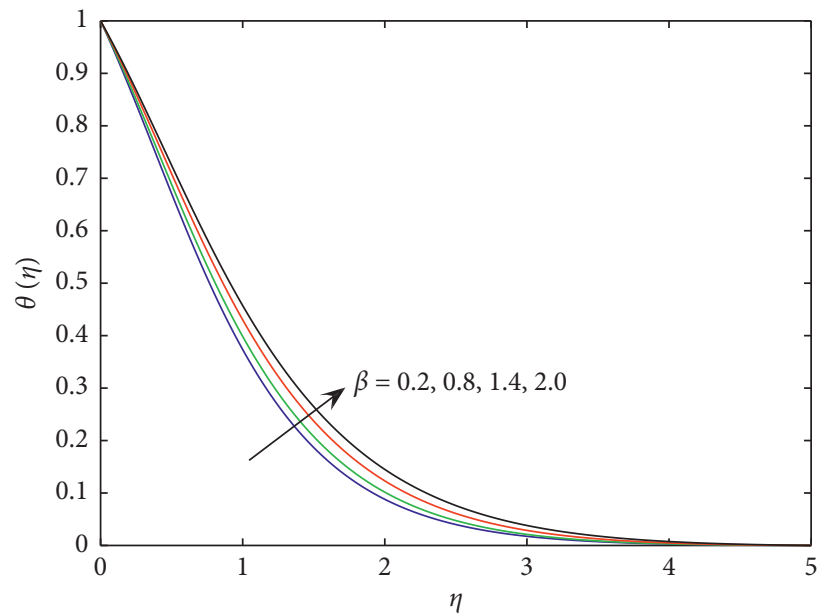

Figure 7: Temperature profile versus $\beta$. 


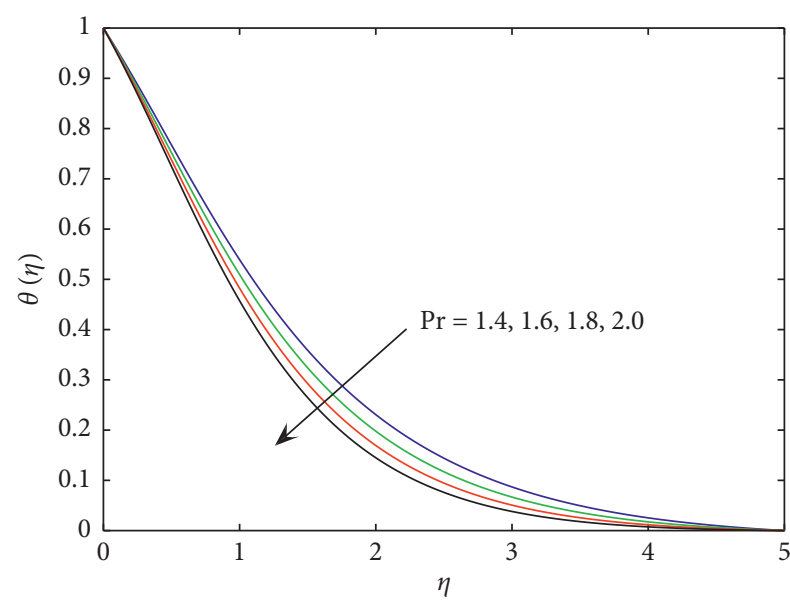

FIgURE 8: Temperature profile versus Pr.

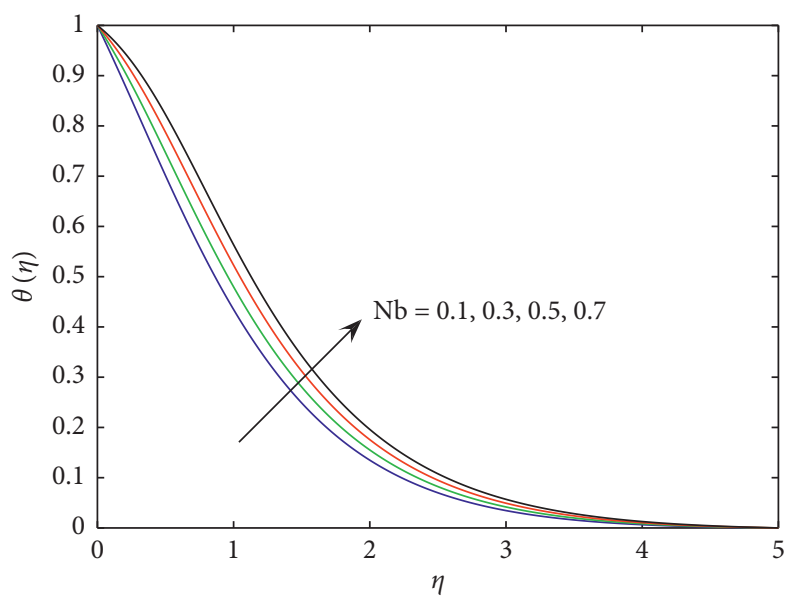

Figure 9: Temperature profile versus $\mathrm{Nb}$.

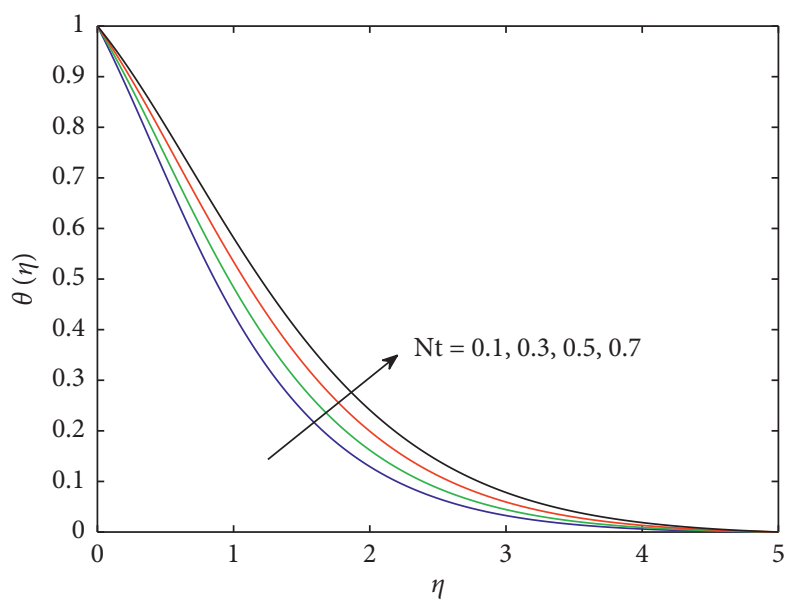

FIgURE 10: Temperature profile versus Nt.

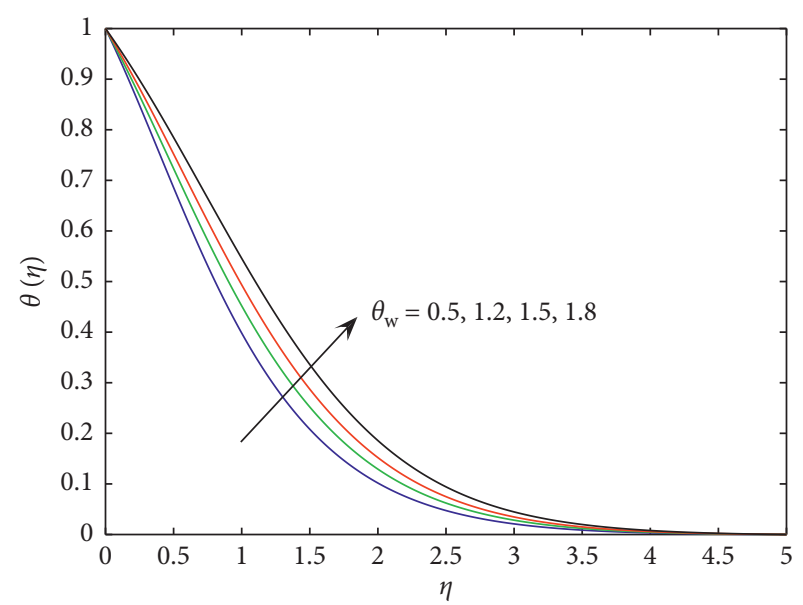

Figure 11: Temperature profile versus $\theta_{w}$.

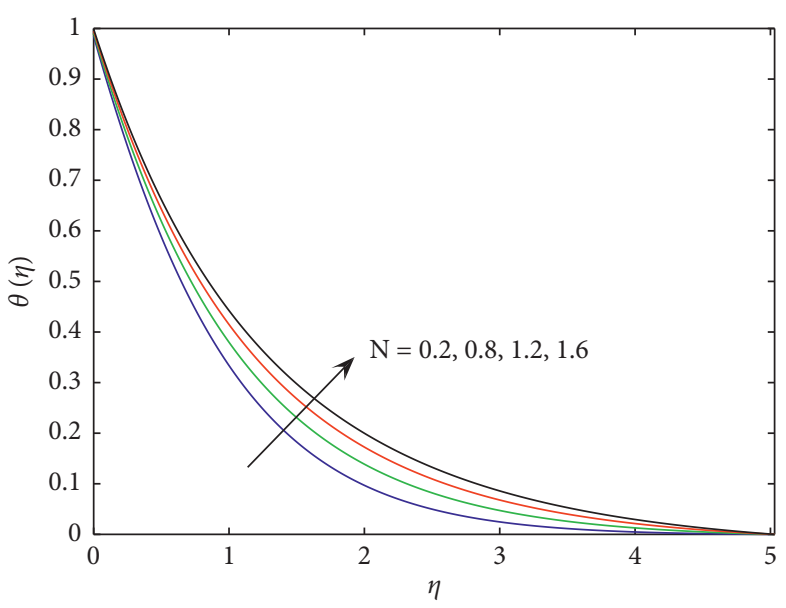

Figure 12: Temperature profile versus $N$.

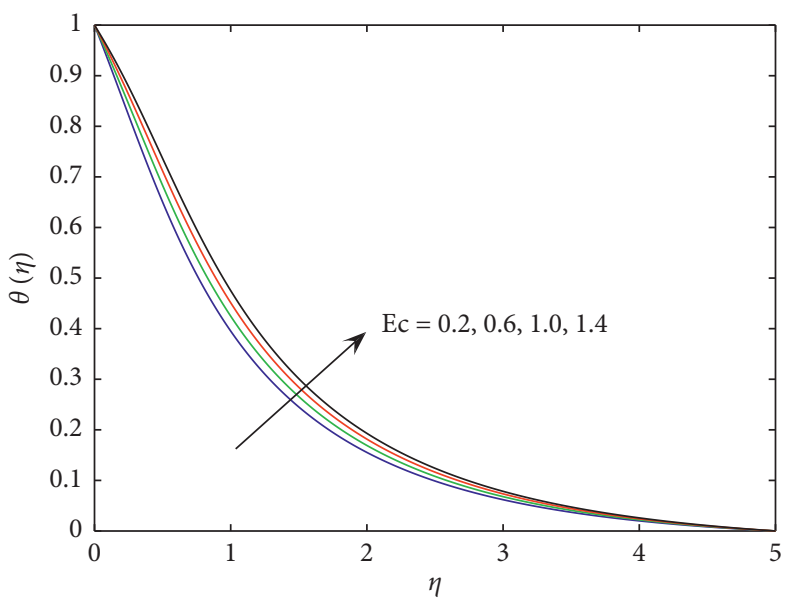

Figure 13: Thermal profile versus Ec. 


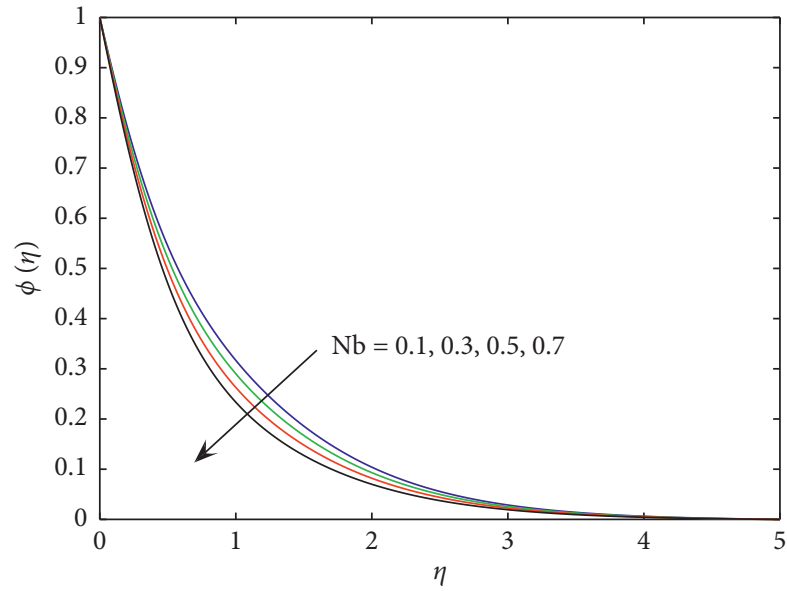

FIgURE 14: Concentration profile versus Nb.

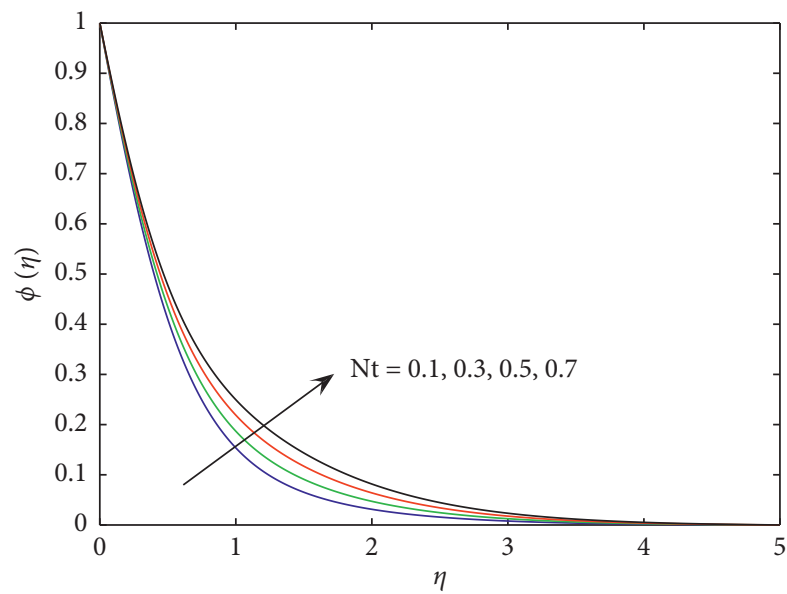

FIGURE 15: Concentration profile versus Nt.

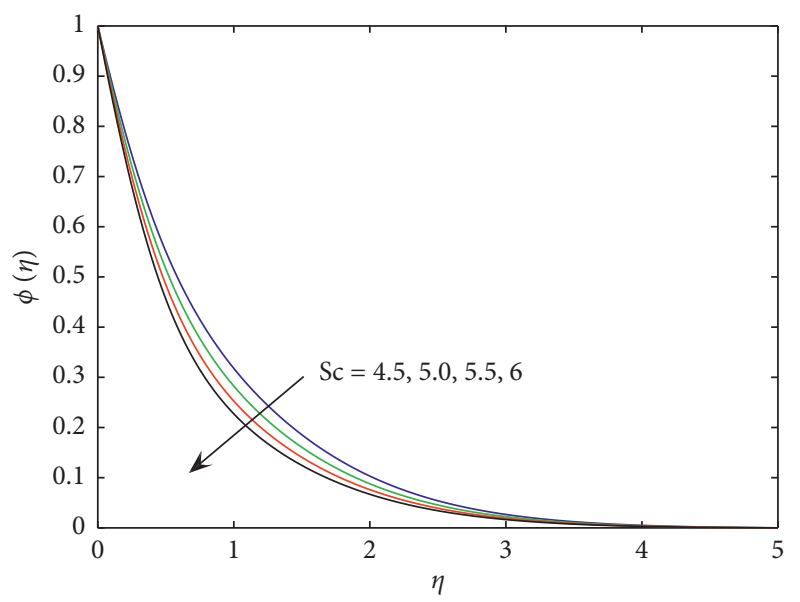

FIgURE 16: Concentration profile versus Sc.

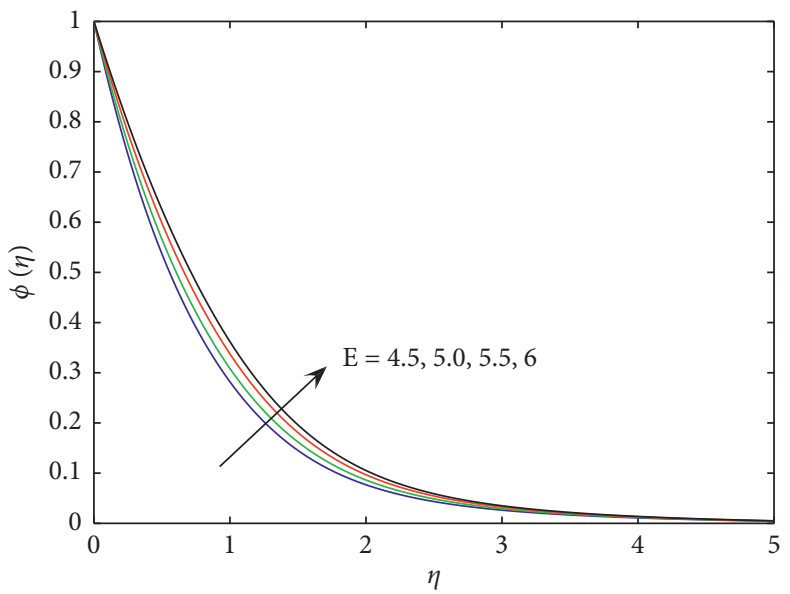

FIgURE 17: Concentration profile versus $E$.

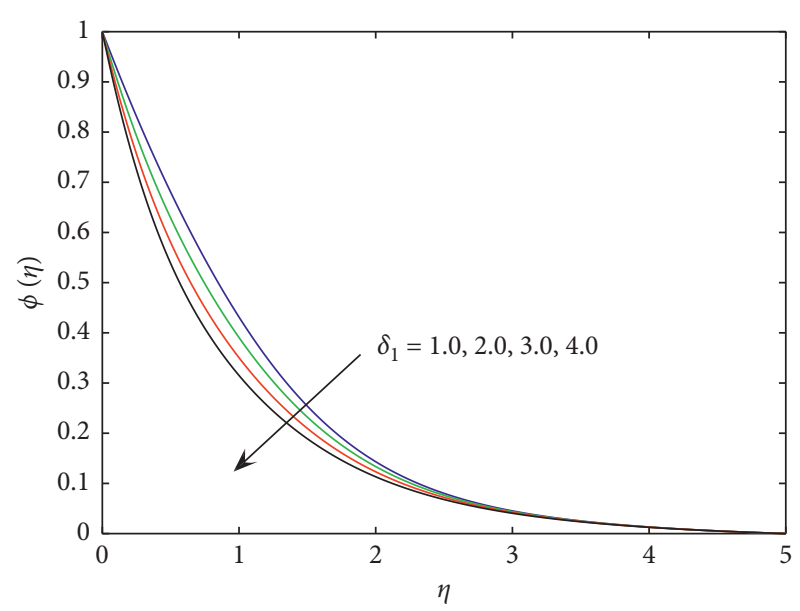

FIgURE 18: Concentration profile versus $\delta_{1}$.

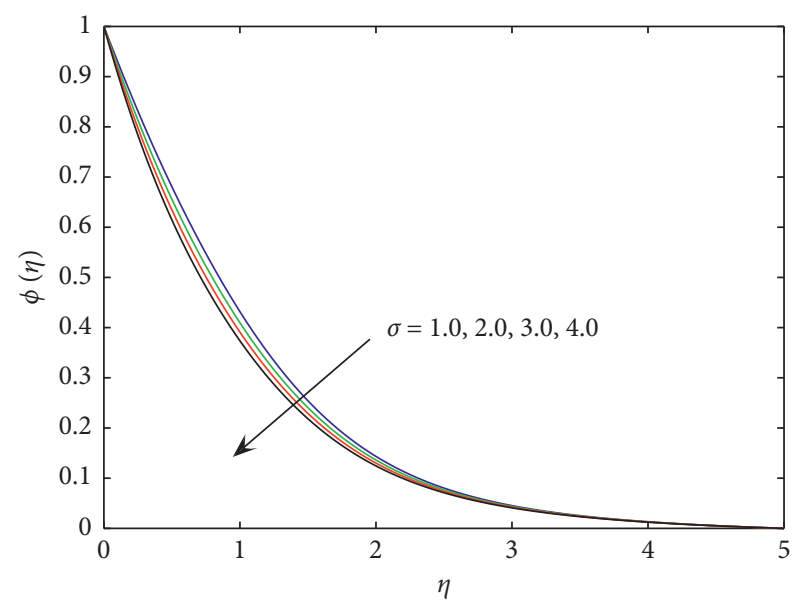

Figure 19: Concentration profile versus $\sigma$. 


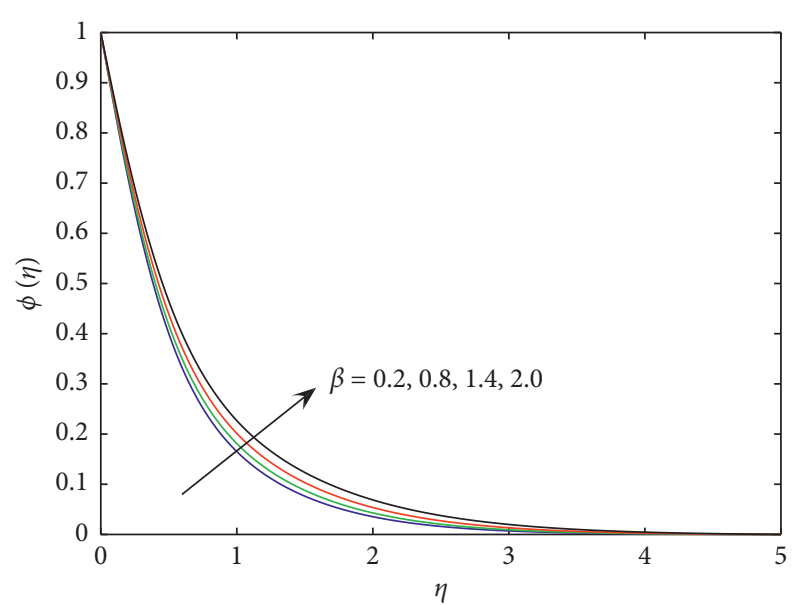

Figure 20: Concentration profile versus $\beta$.

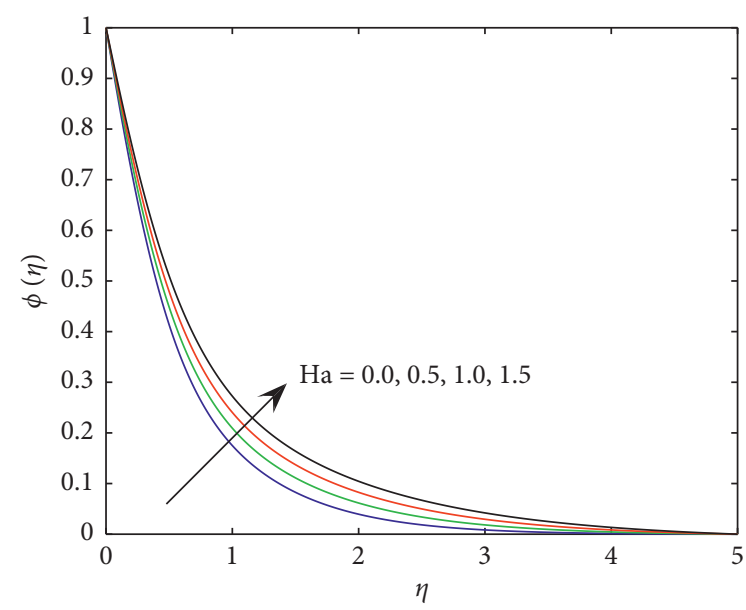

Figure 21: Concentration profile versus $\mathrm{Ha}$.

inversely coupled. So, higher estimation in (Sc) intensively declines the solutal layer and so does the profile.

Figure 17 highlights the physical nature and behaviour of the activation energy parameter $(E)$ within the variational frame against $\phi(\eta)$ nanofluid concentration. One can detect that intensification of $(E)$ parameter enhances the concentration profile. On the base of physical sense, for greater $(E)$ parametric values delineating the basic Arrhenius activation energy factor, it results in boosting up the rate of chemical reaction. This enhancement in the chemical reaction rate intensifies $\phi(\eta)$.

Figure 18 signifies the aspects caused by the discrete variation in the temperature difference parameter $\left(\delta_{1}\right)$ over $\phi(\eta)$. Focusing the graph demonstrates that $\phi(\eta)$ reduced gradually until it vanishes. The physical significance is the escalation in the destructive chemical reaction rate.

Figure 19 reveals the outcomes of the chemical reaction rate $(\sigma)$ parameter that tells the physical interpretation of vanishing the concentration profile as the parametric value varies. By increasing the values of $((\sigma))$, more species are consumed so the rate of chemical reaction increases which causes to decline the concentration profile.
Figure 20 demonstrates the impact, due to fluctuating values of $\beta$ on $\phi(\eta)$, and exhibits that, with uplifting values of $\beta$, there occurs an increment in the concentration profile. Because, for increase in parameter $\beta$, resistive force comes into being that causes an increase in the concentration of nanoparticles in the fluid flow due to which the energy transportation increases and ultimately increases the concentration profile.

Figure 21 poses the effect of the Ha number on the concentration profile. The figure exhibits that, with higher values of the Ha parameter, the concentration profile increases because of enhancement in concentration boundary layer thickness as the Lorentz force causes an opposing reaction in the motion of nanoparticles and enhances the concentration of particle, thereby enhancing the concentration profile.

\section{Concluding Remarks}

This research work summarizes the analysis about viscous and ohmic dissipation effects through a stretchable sheet. Chemical reactions are under consideration. The analysis is deliberately performed in the presence of magnetic dipole. The heat transfer characteristics causes the generation of heat in the presence of magnetic dipole where viscous and ohmic dissipation aspects are reported. Heat generation aspect is preserved. The impact of nonlinear thermal radiation in the flow of ferromagnetic Eyring-Powell nanofluid (a lot of its application involving paper production, glass filaments, etc.) under the magnetic field persuaded by the dipole effects is described in the orientation of the model just because of its wide applications in areas of space technology and aerospace engineering. The aim of this research work is to lessen the drag friction and intensify the rate of heat and mass transfer. The key findings of aforementioned analysis are itemized as

(i) An intensification of $\beta$, which ultimately delineates the nanoliquid velocity profile $f^{\prime}(\eta)$.

(ii) Thermal profile $\theta(\eta)$ of the nanofluid during flow and its corresponding layer thickness enhances via progressive estimation in $\beta, \mathrm{N}, \mathrm{Ec}, \theta_{w}, \gamma, N_{b}, N_{t}$, and $E_{1}$. It can be noted that, with increase in thermal radiation, temperature tends to rise.

(iii) Increasing $\operatorname{Pr}$ causes reduction in the profile $\theta(\eta)$. This scenario is perceived because of weaker diffusivity subject to higher Pr.

(iv) For augmented values of fluid parameter $\varepsilon$ and $\delta$, the velocity gradient, say $C_{f}\left(\operatorname{Re}_{x}\right)^{(1 / 2)}$, has reverse impact.

(v) The thermal gradient $\mathrm{Nu}\left(\mathrm{Re}_{x}\right)^{-(1 / 2)}$ increases for larger $(\mathrm{Pr})$ and $(\mathrm{N})$ parameters, while showing a reverse trend for varying $(\mathrm{Ec})$ and $(\mathrm{Ha})$ parameters.

(vi) The solutal gradient $-\mathrm{Sh}\left(\operatorname{Re}_{x}\right)^{-(1 / 2)}$ increases for larger $(\sigma),(\mathrm{Sc})$, and $\left(\delta_{1}\right)$ parameters, while showing a reverse trend for the varying $(E)$ parameter. 
(vii) $\theta(\eta)$ exhibits a decaying behaviour with respect to incremented values of $\theta_{w}$.

(viii) $E_{1}$, the local electric parameter, casts a positive impact on the velocity profile while thermal profile depreciates throughout the boundary layer.

(ix) Concentration profile $\phi(\eta)$ exhibits a reverse trend for Brownian and thermophoresis variables i.e., decreases for $N_{b}$ and increases for $N_{t}$.

(x) $\phi(\eta)$ rises for $\beta$ and also shows increasing behaviour for larger activation energy $E$.

(xi) Increasing the Hartmann number $(\mathrm{Ha})$ poses to delineate the $f^{\prime}(\eta)$ profile, while showing a reverse behaviour in case of $\theta(\eta)$.

(xii) Larger values of $\delta_{1}$ and $\sigma$ resulted in the decaying nature of $\phi(\eta)$.

(xiii) The behaviour of the Schmidt number (Sc) on the mass transfer process exhibits that for higher values of Sc declines the concentration distribution which results in decreasing the concentration boundary layer thickness.

(xiv) The model orientation transforms into viscous fluid situation if $\varepsilon=\delta=0$

\section{AbbreviationsGreek symbols}

$\begin{array}{ll}\alpha: & \text { Dimensionless distance } \\ \bar{\gamma}: & \text { Shear rate viscosity } \\ \beta: & \text { Ferrohydrodynamic interaction variable } \\ \delta^{*}: & \text { Mean absorption coefficient } \\ \delta_{1}: & \text { Temperature difference parameter } \\ \kappa: & \text { Boltzmann constant } \\ \lambda: & \text { Viscous dissipation factor } \\ \lambda_{0}: & \text { Magnetic permeability } \\ \mu: & \text { Dynamic viscosity } \\ \nu: & \text { Kinematic viscosity } \\ \Phi^{*}: & \text { Magnetic scalar potential } \\ \psi: & \text { Stream function } \\ \rho_{f}: & \text { Base liquid density } \\ \sigma: & \text { Chemical reaction rate constant } \\ \sigma^{*}: & \text { Stefan-Boltzmann constant } \\ \tau_{w}: & \text { Wall shear stress } \\ \theta_{w}: & \text { Temperature ratio variable } \\ \varepsilon \text { and } \delta: & \text { Material parameters } \\ \varepsilon_{1}: & \text { Temperature difference variable. }\end{array}$

\section{Symbols}

$\mathbf{A}_{1}$ : $\quad$ First Rivilin-Ericksen tensor

Q: $\quad$ Extrastress tensor

v: $\quad$ Velocity field

a: $\quad$ Positive constant

$B_{0}: \quad$ Uniform transverse magnetic field

$C$ : Concentration

$c: \quad$ Distance

$C_{f x}: \quad$ Velocity gradient

$c_{p}: \quad$ Specific heat

$D_{B}$ : $\quad$ Brownian diffusion coefficient
$D_{T}: \quad$ Thermophoretic diffusion coefficient

$E: \quad$ Dimensionless activation energy

$E_{0}: \quad$ Electric field

$E_{1}: \quad$ Electric parameter

$E_{a}: \quad$ Activation energy

Ec: $\quad$ Eckert number

$H$ : $\quad$ Magnetic field

Ha: Hartmann number

$k$ : $\quad$ Thermal conductivity

$K_{1}: \quad$ Pyromagnetic coefficient

$k_{r}^{2}: \quad$ Chemical reaction rate constant

M: $\quad$ Magnetization

$N$ : Thermal radiation variable

$n: \quad$ Fitted rate constant

$N_{b}$ : $\quad$ Brownian movement variable

$N_{t}$ : Thermophoresis variable

$\mathrm{Nu}_{x}: \quad$ Thermal gradient

p: $\quad$ Pressure

Pr: $\quad$ Prandtl number

$q_{w}: \quad$ Wall heat flux

$\mathrm{Re}_{x}: \quad$ Local Reynolds number

Sc: $\quad$ Schmidt number

$T$ : $\quad$ Cauchy stress tensor

$T_{\infty}: \quad$ Ambient temperature

$T_{w}$ : $\quad$ Surface temperature

$u$ and $v$ : Velocity components

$u_{w}$ : Wall velocity

$x$ and $y$ : Space coordinates

I: Identity tensor.

\section{Data Availability}

The data used to support the findings of the study are included within the article.

\section{Additional Points}

(i) The effects of viscous and ohmic dissipation under the influence of magnetic dipole for Eyring-Powell nanofluid are studied. (ii) The Buongiorno model is taken into account while considering the stretchable surface. (iii) The impacts of thermal radiation, thermophoresis, and Brownian movement parameters are observed for the respective nanofluid. (iv) The varying Schmidt number and chemical reaction lessen the thickness of concentration profile. (v) Graphical behavior is presented for each of the affecting physical parameter with tabular description of the skin friction coefficient, Nusselt number, and Sherwood number.

\section{Conflicts of Interest}

The authors declare that they have no conflicts of interest.

\section{References}

[1] L. J. Crane, "Flow past a stretching plate," Zeitschrift für Angewandte Mathematik und Physik ZAMP, vol. 21, no. 4, pp. 645-647, 1970.

[2] K. B. Pavlov, "Magnetohydrodynamic flow of an incompressible viscous fluid caused by deformation of a plane 
surface," Magnitnaya Gidrodinamika, vol. 4, no. 1, pp. 146148, 1974.

[3] P. S. Gupta and A. S. Gupta, "Heat and mass transfer on a stretching sheet with suction or blowing," Canadian Journal of Chemical Engineering, vol. 55, no. 6, pp. 744-746, 1977.

[4] G. Rasool, A. Shafiq, M. S. Alqarni, A. Wakif, I. Khan, and M. S. Bhutta, "Numerical scrutinization of Darcy-forchheimer relation in convective magnetohydrodynamic nanofluid flow bounded by nonlinear stretching surface in the perspective of heat and mass transfer," Micromachines, vol. 12, no. 4, p. 374, 2021.

[5] H. J. Kroh and B. U. Felderhof, "Electromagnetodynamics of polar liquids and suspensions," Zeitschrift fur Physik B Condensed Matter, vol. 66, no. 1, pp. 1-6, 1987.

[6] S. U. Choi and J. A. Eastman, "Enhancing thermal conductivity of fluids with nanoparticles (No. ANL/MSD/CP-84938; CONF951135-29)," Argonne National Lab, Lemont, IL USA, 1995.

[7] G. Rasool and A. Wakif, "Numerical spectral examination of EMHD mixed convective flow of second-grade nanofluid towards a vertical Riga plate using an advanced version of the revised Buongiorno's nanofluid model," Journal of Thermal Analysis and Calorimetry, vol. 143, pp. 2379-2393, 2021.

[8] A. Rehman, S. Achakzai, S. Nadeem, and S. Iqbal, "Stagnation point flow of eyring-powell fluid in a vertical cylinder with heat transfer," Journal of Power Technologies, vol. 96, no. 1, pp. 57-62, 2016.

[9] T. M. Healy, C. Lucas, and A. P. Yoganathan, "Noninvasive fluid dynamic power loss assessments for total cavopulmonary connections using the viscous dissipation function: a feasibility study," Journal of Biomechanical Engineering, vol. 123, no. 4, pp. 317-324, 2001.

[10] V. R. N. Telis, J. Telis-Romero, H. B. Mazzotti, and A. L. Gabas, "Viscosity of aqueous carbohydrate solutions at different temperatures and concentrations," International Journal of Food Properties, vol. 10, no. 1, pp. 185-195, 2007.

[11] M. Eslamian and M. Z. Saghir, "A critical review of thermodiffusion models: role and significance of the heat of transport and the activation energy of viscous flow," Journal of Non-Equilibrium Thermodynamics, vol. 34, no. 2, pp. 97-131, 2019.

[12] J. S. Babu and S. P. Sathian, "The role of activation energy and reduced viscosity on the enhancement of water flow through carbon nanotubes," The Journal of Chemical Physics, vol. 134, no. 19, Article ID 194509, 2011.

[13] T. Javed, N. Ali, Z. Abbas, and M. Sajid, "Flow of an EyringPowell non-Newtonian fluid over a stretching sheet," Chemical Engineering Communications, vol. 200, no. 3, pp. 327-336, 2013.

[14] T. Hayat, T. Muhammad, A. Alsaedi, and M. S. Alhuthali, "Magnetohydrodynamic three-dimensional flow of viscoelastic nanofluid in the presence of nonlinear thermal radiation," Journal of Magnetism and Magnetic Materials, vol. 385, pp. 222-229, 2015.

[15] P. V. Satya Narayana and D. Harish Babu, "Numerical study of MHD heat and mass transfer of a Jeffrey fluid over a stretching sheet with chemical reaction and thermal radiation," Journal of the Taiwan Institute of Chemical Engineers, vol. 59, pp. 18-25, 2016.

[16] A. Zeeshan and A. Majeed, "Effect of magnetic dipole on radiative non-Darcian mixed convective flow over a stretching sheet in porous medium," Journal of Nanofluids, vol. 5, no. 4, pp. 617-626, 2016.

[17] A. Zeeshan and A. Majeed, "Non Darcy mixed convection flow of magnetic fluid over a permeable stretching sheet with ohmic dissipation," Journal of Magnetics, vol. 21, no. 1, pp. 153-158, 2016.

[18] T. Gul, A. Khan, M. Bilal et al., "Magnetic dipole impact on the hybrid nanofluid flow over an extending surface," Scientific Reports, vol. 10, no. 1, p. 8474, 2020.

[19] K. Arshad, S. Anwar, T. Asifa et al., "Bio-convective micropolar nanofluid flow over thin moving needle subject to Arrhenius activation energy, viscous dissipation and binary chemical reaction," Case Studies in Thermal Engineering, vol. 25, Article ID 100989, 2021.

[20] S. M. Hussain, J. Jain, G. S. Seth, and M. M. Rashidi, "Free convective heat transfer with hall effects, heat absorption and chemical reaction over an accelerated moving plate in a rotating system," Journal of Magnetism and Magnetic Materials, vol. 422, pp. 112-123, 2017.

[21] R. Mahmood, N. Kousar, M. Yaqub, and K. Jabeen, "Numerical simulations of the square lid driven cavity flow of bingham fluids using nonconforming finite elements coupled with a direct solver," Advances in Mathematical Physics, vol. 2017, Article ID 5210708, 10 pages, 2017.

[22] E. Azhar, Z. Iqbal, and E. N. Maraj, "Viscous dissipation performance on stagnation point flow of Jeffrey fluid inspired by internal heat generation and chemical reaction," Thermal Science and Engineering Progress, vol. 13, pp. 100-377, 2019.

[23] K. Jabeen, M. Mushtaq, and R. M. A. Muntazir, "A comparative study of MHD flow analysis in a porous medium by using differential transformation method and variational iteration method," Journal of Contemporary Applied Mathematics, vol. 9, no. 2, pp. 2222-5498, 2019.

[24] M. Waqas and S. Jabeen, "Numerical simulation for nonlinear radiated Eyring-Powell nanofluid considering magnetic dipole and activation energy," International Communications in Heat and Mass Transfer, vol. 112, pp. 104-401, 2020.

[25] K. Jabeen, M. Mushtaq, and R. M. A. Muntazir, "Analysis of MHD fluids around a linearly stretching sheet in porous media with thermophoresis, radiation, and chemical reaction," Mathematical Problems in Engineering, vol. 2020, Article ID 9685482, 14 pages, 2020.

[26] K. Jabeen, M. Mushtaq, and R. M. A. Muntazir, "Analysis of the MHD boundary layer flow over a nonlinear stretching sheet in a porous medium using semianalytical approaches," Mathematical Problems in Engineering, vol. 2020, Article ID 3012854, 9 pages, 2020.

[27] R. M. A. Muntazir, K. Jabeen, and M. Mushtaq, "A numerical study of MHD carreau nanofluid flow with gyrotactic microorganisms over a plate, wedge, and stagnation point," Mathematical Problems in Engineering, vol. 2021, Article ID 5520780, 22 pages, 2021.

[28] R. M. A. Muntazir, K. Jabeen, M. Mushtaq, and S. Shahzadi, "Influence of chemically reacting ferromagnetic carreau nanofluid over a stretched sheet with magnetic dipole and viscous dissipation," Mathematical Problems in Engineering, vol. 2021, Article ID 6652522, 12 pages, 2021.

[29] R. M. A. Muntazir, K. Jabeen, M. Mushtaq, and S. Shahzadi, "A study of MHD nanofluid flow around a permeable stretching sheet with thermal radiation and viscous dissipation," Proceedings of the Institution of Mechanical EngineersPart C: Journal of Mechanical Engineering Science, (Accepted), 2021.

[30] K. Jabeen, M. Mushtaq, and R. M. A. Muntazir, "Suction and injection impacts on casson nanofluid with gyrotactic microorganisms over a moving wedge," ASME: Journal of Fluids Engineering, 2021, Accepted. 